TI 2011-154/4

Tinbergen Institute Discussion Paper

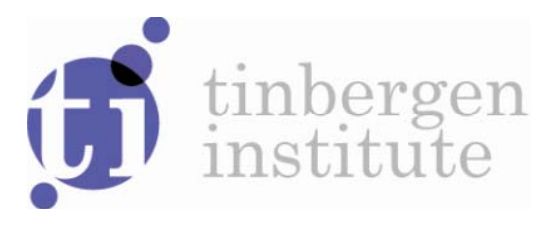

\title{
Measuring and Predicting Heterogeneous Recessions
}

Cem Cakmaklir

Richard Paap²

Dick van Dijk²

' Faculty of Economics and Business, University of Amsterdam;

2 Erasmus School of Economics, Erasmus University Rotterdam, and Tinbergen Institute. 
Tinbergen Institute is the graduate school and research institute in economics of Erasmus University Rotterdam, the University of Amsterdam and VU University Amsterdam.

More TI discussion papers can be downloaded at http://www.tinbergen.nl

Tinbergen Institute has two locations:

Tinbergen Institute Amsterdam

Gustav Mahlerplein 117

1082 MS Amsterdam

The Netherlands

Tel.: +31(0)205251600

Tinbergen Institute Rotterdam

Burg. Oudlaan 50

3062 PA Rotterdam

The Netherlands

Tel.: +31(0)10 4088900

Fax: $+31(0) 104089031$

Duisenberg school of finance is a collaboration of the Dutch financial sector and universities, with the ambition to support innovative research and offer top quality academic education in core areas of finance.

DSF research papers can be downloaded at: http://www.dsf.nl/

Duisenberg school of finance

Gustav Mahlerplein 117

1082 MS Amsterdam

The Netherlands

Tel.: +31(0)20 5258579 


\title{
Measuring and Predicting Heterogeneous Recessions *
}

\author{
Cem Çakmaklı ${ }^{\dagger, 1}$ Richard Paap ${ }^{\ddagger} 2 \quad$ Dick van Dijk ${ }^{\S, 2}$ \\ ${ }^{1}$ Department of Quantitative Economics, University of Amsterdam \\ ${ }^{2}$ Econometric Institute, Erasmus University Rotterdam
}

\section{October 2011}

\begin{abstract}
This paper conducts an empirical analysis of the heterogeneity of recessions in monthly U.S. coincident and leading indicator variables. Univariate Markovswitching models indicate that it is appropriate to allow for two distinct recession regimes, corresponding with 'mild' and 'severe' recessions. All downturns start with a mild decline in the level of economic activity. Contractions that develop into severe recessions mostly correspond with periods of substantial credit squeezes as suggested by the 'financial accelerator' theory. Multivariate Markov-switching models that allow for phase shifts between the cyclical regimes of industrial production and the Conference Board Leading Economic Index confirm these findings.
\end{abstract}

Keywords: Business cycle, phase shifts, regime-switching models, Bayesian analysis

JEL Classification: $C 11, C 32, C 51, C 52$, E32

\footnotetext{
*We would like to thank Paolo Giordani, Mark Jensen, Gary Koop, John Maheu, Hashem Pesaran and Rodney Strachan for helpful comments and suggestions. We would also like to thank conference participants at the 2nd Conference on Recent Developments in Macroeconomics (ZEW, Mannheim, June 2010), the Rimini Conference in Economics and Finance (RCEF'10; Rimini, June 2010) and seminar participants at the Tinbergen Institute and the Helsinki Center of Economic Research. Any remaining errors are our own.

${ }^{\dagger}$ Correspondence to: Cem Çakmakl, Valckenierstraat 65-67, 1018 XE Amsterdam, The Netherlands, e-mail: C.Cakmakli@uva.nl.

${ }^{\ddagger}$ P.O. Box 1738, NL-3000 DR Rotterdam, The Netherlands, e-mail: paap@ese.eur.nl.

§P.O. Box 1738, NL-3000 DR Rotterdam, The Netherlands, e-mail: djvandijk@ese.eur.nl.
} 


\section{Introduction}

A century of empirical evidence about the US business cycle has revealed that economic recessions come in a variety of shapes and sizes. While some downturns are relatively mild, others are rather severe. While some recessions are short, lasting only about half a year, the duration of others is substantially longer. While some contractions are followed by rapid and strong recoveries of economic activity, the effects of other recessions are felt much longer after they have ended. Interestingly, the recent recession, often labeled as the 'Great Recession', seems to have combined the worst of these different dimensions, in the sense that it was long lasting (from December 2007 until June 2009, according to the NBER business cycle dating committee), severe (with an average annualized quarterly GDP growth rate of $-3.5 \%$ between 2008Q1 and 2009Q2), and followed by a sluggish recovery.

Not surprisingly, a substantial body of research has been devoted to understanding the characteristics and dynamics of recessions. One of the key points of interest in this literature has been the determinants of the severity of recessions, dating back at least to Fisher (1933). Motivated mostly by the 'Great Depression' during 1929-1933, several theories on the dynamics of recessions have been put forward specifically addressing the question why some recessions turn out to be more severe than others. Mishkin (1978), Bernanke (1981), Romer (1993), Bernanke et al. (1996, 1999), and Gertler and Kiyotaki (2010), among others, discuss several mechanisms and channels having adverse effects on output and aggregate demand during recessions. These include the lack of financial intermediation, the uncertainty brought about by stock market crashes changing consumer preferences, and the deteriorated household balance-sheets caused by increasing real burdens due to deflationary periods during recessions, among others. A common theme in these theories is that

recessions start with mild negative shocks but different mechanisms (such as the credit channel, the uncertainty channel, the balance sheet channel, and so forth) can 
amplify their effects or trigger more severe shocks and, hence, affect the severity of the recession.

Motivated by both empirical evidence and theory, in this paper we statistically analyze the relevance of distinguishing different types of economic downturns. Applying Markov-Switching (Vector) AutoRegressive (MS-(V)AR) models, we examine whether it is useful to discriminate between mild and severe recessions in monthly coincident and leading indicator variables. Our analysis consists of three parts. We first conduct a univariate analysis on the Conference Board Coincident Economic Index (CEI) and its four constituents (the number of employees on non-agricultural payrolls (ENP), personal income less transfer payments (PI), industrial production (IP) and manufacturing and trade sales (MTS)) as well as the Conference Board Leading Economic Index (LEI). By analyzing both individual coincident variables as well as the CEI, we aim to uncover the characteristics of regimes each individual variable embeds and to explore the effects of aggregating individual variables to a single index on the regime switching dynamics. Second, we employ a multivariate analysis using a coincident variable that represents the business cycle most accurately together with the LEI. Specifically, we use IP for that purpose, as it appears from our univariate analysis that this variable captures the NBER recessions and expansions most closely. Third and finally, we attempt to link our empirical findings to the theories concerning the determinants of the severity of recessions. In particular, we document empirical evidence in favor of the 'financial accelerator' theory (Bernanke et al., 1996, 1999), by showing a link between the behavior of credit spreads and the severity of recessions.

Our univariate analysis shows that a three regime MS-AR model is most appropriate for most of the individual business cycle indicators as well as for the CEI and the LEI. In most of the cases the regimes may be characterized as expansions, mild recessions and severe recessions. This finding contrasts most of the previous 
studies that apply Markov (and other types of) regime-switching models in this context, which typically find that the third regime (beyond expansions and recessions) captures a recovery or 'bounce back' phase, see Sichel (1994), Boldin (1996), and Clements and Krolzig (2003), among others. A notable exception is Hamilton (2005), who reports a three regime Markov-switching model for the postwar unemployment rate also with separate regimes for mild and severe recessions. For the CEI, we find that the two types of recessions actually correspond with recessions before and after the mid 1980s. This distinction, however, is mostly due to the occurrence of slow recoveries following the more recent recessions, leading to rather prolonged recession signals in the early 1990s and 2000s (as these mild recoveries are considered as part of the recessions), which is at odds with the NBER business cycle chronology. This effect mostly arises because of the sluggish improvement in labor market conditions (hence the name 'jobless recoveries', see Gordon (1993); Groshen and Potter (2003)), which is confirmed by the fact that we find the same phenomenon for employment. On the other hand, the three regime model for industrial production captures both mild and severe recessions successfully and produces signals very close to the NBER recession dates. The posterior probabilities reveal three severe recession periods, occurring during the recessions of 1974-5, 1980 and 2007-9. A three regime model of LEI results in similar findings.

In our multivariate analysis, we use the MS-VAR model developed in Paap et al. (2009) and Cakmakli et al. (2011). This model has two attractive features. First, by employing a multivariate model of similar variables it provides more precise regime signals and better predictions. Second, as the model identifies the degree of synchronization of the cycles in the IP and the LEI it allows us to assess the LEI's ability to predict the severity of recessions. Here, by synchronization we mean that the different variables in fact share a single common cyclical component but subject to different phase shifts, where the lead-lag time can differ across regimes. We show 
that this type of MS-VAR model with three regimes describes the US business cycle better than the models with two regimes. Results indicate that LEI is most timely in predicting moderate recessions with a lead time of 12 months, while the lead time of severe recessions is (only) 6 months.

It is useful to note that in the context of two-regime Markov-switching models several specifications have been proposed to capture different recession shapes. Hamilton (1989)'s original formulation with regime-switching mean growth rates and homoskedastic errors implies that recessions are so-called 'L'-shaped, as there is no subsequent fast-growth recovery phase. Kim et al. (2005) augment Hamilton's original model with a so-called 'bounce-back' term, such faster growth in the quarters immediately following a recession can be generated. The strength of the recovery can be linked to the length of the preceding recession or its depth, or both. Depending on the exact specification, the model can capture 'V'-shaped recessions, characterized by a strong recovery after a sharp contraction, or 'U'-shaped recessions with a smoother transition from contractions to expansions. Morley and Piger (forthcoming) provide an excellent recent comparison of these bounce-back specifications. While the bounce-back approach has intuitive appeal, we do not adopt it in this paper. Note that it focuses on the properties of the post-recession period, and its relation with the preceding recessions. The recessions themselves, however, are assumed to be all identical, in the sense that the recession regime is characterized by a constant mean growth rate, independent of the length or severity of the ongoing recession (or of anything else). Given that the main purpose of our analysis is to explore whether it is useful to distinguish different types of recessions, we consider Markov-switching models with multiple, unrestricted regimes.

Besides the stimulating effects of lower price levels and interest rates on consumption (wealth effect) as well as on investment (Keynes effect) during recessions, several mechanisms have been put forward that may lead to the deepening of a 
contraction (and hence the occurrence of a severe recession). Mishkin (1978) emphasizes the effect of deflation on aggregate demand during the Great Depression, related to the increasing real debt burden of households. This caused consumers to cut their spending on illiquid assets (durable goods, residential housing assets, and so forth) and/or to liquidation, thereby shifting household's balance-sheet toward liquidity. Considering the possible relation between the Great Crash in 1929 and the subsequent Great Depression, Romer (1990) points out the link between uncertainty and consumption decisions. The stock market crash in 1929 and the resulting long-lasting extreme variation in stock prices increased uncertainty for consumers about their future income stream. This, in general, caused people to defer their irreversible, durable goods consumption and to increase consumption of reversible (nondurable) goods. Bernanke et al. $(1996,1999)$ focus on the 'small shocks, large cycles' puzzle in their 'financial accelerator' theory. Referring to the amplification of initial shocks brought about by changes in credit-market conditions (the 'financial accelerator'), this theory suggests that first, borrowers facing relatively high agency costs in credit markets will bear the brunt of economic downturns (due to the fact that many investors attempt to move their money into relatively safe investments, i.e. the flight to quality). Second, reduced spending, production, and investment by high-agency-cost borrowers will exacerbate the effects of recessionary shocks. We document empirical evidence in favor of the financial accelerator theory by showing a link between the behavior of credit spreads and the severity of recessions.

The remainder of the paper is organized as follows. We first provide a description of the data and provide a preliminary analysis in Section 2. We describe the univariate Markov-switching AR model and discuss our univariate empirical findings in Section 3. In Section 4, we discuss the Markov-switching VAR model with different phase shifts for multiple regimes. We also provide the multivariate empirical results in this section. We document the link between the behavior of credit spreads and 
the severity of recessions in Section 5. Finally, we conclude in Section 6. Technical details are deferred to a set of appendices.

\section{Stylized facts of US recessions}

We analyze the Conference Board Coincident Economic Index (CEI), its four components, and the Conference Board Leading Economic Index (LEI). The CEI is composed of the number of employees on non-agricultural payrolls (ENP), personal income less transfer payments (PI), industrial production (IP) and manufacturing and trade sales (MTS). Monthly observations for all variables are available for the period from January 1960 until October 2010.

Figure 1 shows the average growth rates of the CEI and its four components during the course of the recessions that occurred during our sample period and their aftermath, see for example Sichel (1994) for a similar analysis. The graphs show the average growth rate in different quarters of recessions as defined by the NBER turning points, and during six-month periods of expansions. The graphs indicate that for the CEI, ENP and IP recessions progressively become more severe, in the sense that their average growth rates monotonically decline during the different quarters of the contraction periods. Note that this goes against the conventional wisdom that recessions typically start with a sharp downturn. While this sort of pattern in the first recession quarter does seem to be present for PI and MTS, we also observe large negative growth rates during the fourth and fifth quarters of recessions for those variables. Hence, contraction periods that last relatively long (i.e. more than three quarters) have a severe latter part also for PI and MTS.

For expansions, we do not observe a uniform pattern across variables. Only MTS follows a pattern consistent with high growth recoveries following recessions. The average growth rate during the first six months of expansions is equal to 0.75 percent, which gradually declines as the expansion continues. IP displays similar behavior, 
Figure 1: Average growth rates of coincident economic indicators over the course of recessions and expansions during the period January 1960 - October 2010
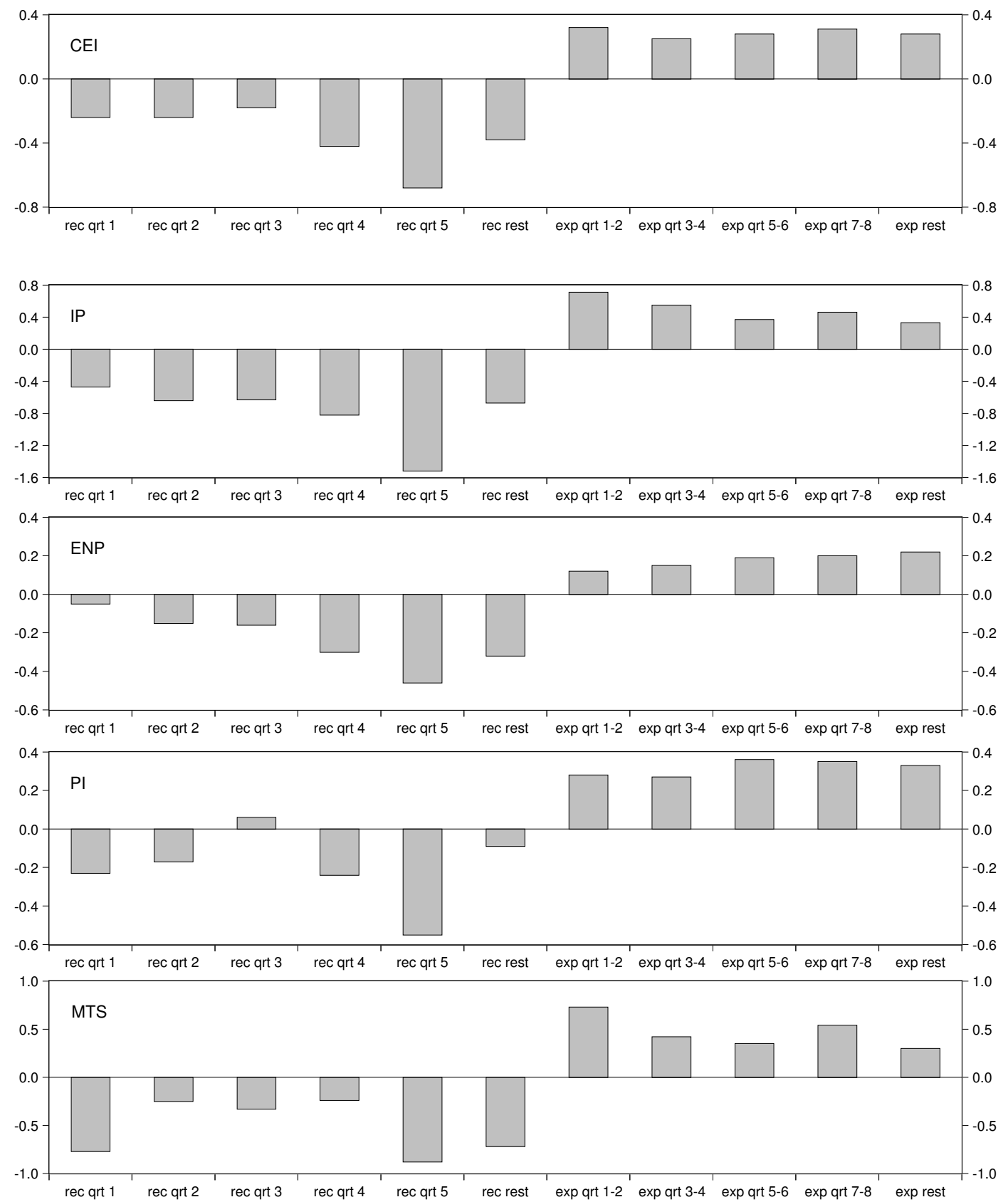

Note: 'rec qrt $j$ ', $j=1, \ldots, 5$, denotes the $j$-th quarter of recessions, while 'rec rest' denotes the remaining periods of recessions. 'exp qrt $2 j-1-2 j$ ', $j=1, \ldots, 4$, denotes quarters $2 j-1$ and $2 j$ of expansions, while 'exp rest' denotes the remaining periods of expansions. 
albeit much less pronounced. For ENP the pattern is reversed in the sense that average growth rate gradually increases during expansions periods. This indicates the sluggish adjustment process of the employment, which was particularly evident during the periods following the 1990-1 and 2001 recessions. When constructing the CEI it seems that these opposite patterns in the individual coincident variables are averaged out to a large extent, such that the average growth rate is approximately the same for all six-month subperiods of expansions.

\section{Univariate analysis}

We analyze the business cycle dynamics in the individual variables by means of univariate Markov-Switching AutoRegressive (MS-AR) models. Our aim is to determine the number of regimes we should distinguish in order to adequately describe the cyclical features in these series, as well as the corresponding regime characteristics.

Let $y_{t}$ denotes the growth rate of a given coincident or leading indicator in month $t$. We assume that the business cycle can be divided into $J$ phases or regimes, which are characterized by different means of $y_{t}$. Unexpected growth, denoted by $\varepsilon_{t}$, is assumed to be normally distributed with time-varying volatility $\sigma_{t}$. The exact specification of the volatility dynamics is described in detail below. We assume that autoregressive coefficients are constant across regimes. ${ }^{1}$ In case of first-order autoregressive dynamics, our assumptions imply the model specification

$$
y_{t}-\mu_{S_{t}}=\phi\left(y_{t-1}-\mu_{S_{t-1}}\right)+\varepsilon_{t} \quad \text { with } \varepsilon_{t} \sim N I D\left(0, \sigma_{t}^{2}\right)
$$

where $S_{t}$ is latent multinomial variable taking the value $j$ if $y_{t}$ is in regime $j$ in month

\footnotetext{
${ }^{1}$ Extending the model to allow for regime-dependent autoregressive dynamics is straightforward. This is not pursued here to keep the complexity of the model at a feasible level. Notice that regime dependence in the mean growth rate already provides a great deal of flexibility.
} 
$t$, and $\mu_{S_{t}}=\mathrm{E}\left[y_{t} \mid S_{t}\right]$ denotes the unconditional mean of $y_{t}$ in the regime indicated by $S_{t}$. The regime-indicator variable $S_{t}$ is assumed to be a first-order homogenous Markov process with transition probabilities

$$
\operatorname{Pr}\left(S_{t}=j \mid S_{t-1}=i\right)=p_{i j} \quad \text { for } i, j=1, \ldots, J .
$$

As one of our aims is to assess the usefulness of additional regimes (on top of expansion and recession phases) to characterize the business cycle dynamics, the specification of the error variance $\sigma_{t}^{2}$ in (1) is not innocuous. Specifically, ignoring regime-switching behavior in the variance may spuriously suggest that multiple regimes are present. For example, assume the business cycle is characterized by two regimes that not only have different mean growth rates but also different variances. Imposing homoskedasticity, we may then find that it is necessary to allow for a third regime, essentially approximating the high volatility regime with two regimes with different mean growth rates. To avoid this issue we consider two possibilities for the time-varying volatility $\sigma_{t}$ in (1). In the first specification, we assume the volatility to be constant across regimes, while in the second we allow for regimedependent heteroskedasticity. In both cases we incorporate a single structural break in the volatilities, to accommodate the Great Moderation, that is, the large and persistent decline in macroeconomic volatility in the mid-1980s, see McConnell and Perez-Quiros (2000), among others. In case of regime-dependent heteroskedasticity we keep the volatility specification parsimonious by imposing that the proportional change in volatility at the time of the structural break is the same in all regimes. This corresponds with the specification

$$
\sigma_{t}=(\delta I[t<\tau]+I[t \geq \tau]) \sigma_{S_{t}}
$$

where $I[A]$ in an indicator function for the event $A, \tau$ is the period when the struc- 
tural break in volatility occurs, and $\delta$ gives the ratio of the volatilities before and after the break. The homoskedastic specification boils down to imposing $\sigma_{S_{t}}=\sigma$ in (3).

\subsection{Estimation and model selection}

We use a Bayesian approach for estimation and inference in the MS-AR model using Markov Chain Monte Carlo (MCMC) techniques. Specifically, we use Gibbs sampling together with data augmentation (see Geman and Geman, 1984; Tanner and Wong, 1987) to obtain posterior results. The estimation of MS-AR models with Bayesian techniques has become common practice in business cycle analysis. Therefore we do not provide a detailed exposition of the estimation procedure here, but we refer to Krolzig (1997) and Kim and Nelson (1999), among others. A description of the estimation of the variance process with a structural break, as specified in (3), is provided in the Appendix A.3 for the multivariate setting considered in the next section.

A few details concerning our Bayesian estimation procedure are useful to note. First, we use noninformative priors for all model parameters, in order to let the data decide on the most appropriate specification. Second, we use the prior for the regime-dependent mean parameters to identify the regimes. Specifically, we set the prior specification for $\mu=\left(\mu_{1}, \mu_{2}, \ldots, \mu_{J}\right)^{\prime}$ as

$$
f(\mu) \propto \begin{cases}1 & \text { if } \mu \in\left\{\mu \in \mathbb{R}^{J} \mid \mu_{1}<\ldots<\mu_{j}<\ldots<\mu_{J}\right\} \\ 0 & \text { elsewhere. }\end{cases}
$$

Note that this prior only involves inequality restrictions and does not impose a particular sign on any of the mean growth rates. Hence, we only rank order the regimes in terms of their mean growth rates but do not enforce any specific type of 
regimes such as recovery or severe recession to be present a priori.

For each of the variables we estimate models with two, three and four regimes with the two different variance specifications. Each model is estimated without and with first-order autoregressive dynamics to account for possible autocorrelation that cannot be captured by the Markov-switching mean (and volatility).

For selecting the most appropriate model specification, we use several criteria. First, we consider the predictive likelihoods of the models. To compute these we first estimate the model parameters using only part of the full sample (denoted as 'estimation sample') and then based on these estimates we evaluate the likelihood of the remaining part. This approach has the advantage that it is not affected by the choice of prior distributions and over-fitting, while it is directly related to the posterior model probabilities, see Geweke and Amisano (2010, 2007). We use the sample from January 1960 until December 2000 for estimation of the model and the remaining period from January 2001 until October 2010 to compute the predictive likelihood. Computational details are provided in Appendix B.

Second, we examine the robustness and stability of the models by comparing the estimation results based upon the initial estimation sample and the full sample.

Third, we check the ability of the models to produce a reasonable description of the business cycle. Using the NBER turning points to define recessions and expansions, we check the compatibility of the posterior regime probabilities with this chronology.

\subsection{Empirical Results}

The estimation results reveal very similar conclusions for three variables, namely CEI, IP and LEI. The results for ENP are also comparable except for the dating of turning points, in particular troughs. Due to the presence of several post-recession periods with sluggish recovery in labor market conditions, the MS-AR models for 
employment contain rather long recessions, ending considerably later than the NBER trough dates. The models for MTS and PI show much less correspondence with the NBER recession dates such that these variables seem to have their own regimeswitching dynamics. Therefore, we provide only detailed results of CEI, IP and LEI for the sake of brevity. ${ }^{2}$

Table 1: Comparison of univariate MS-AR models: Log predictive likelihood values

\begin{tabular}{|c|c|c|c|c|c|c|}
\hline & \multicolumn{3}{|c|}{$\operatorname{AR}(0)$} & \multicolumn{3}{|c|}{$\mathrm{AR}(1)$} \\
\hline & 2 Regimes & 3 Regimes & 4 Regimes & 2 Regimes & 3 Regimes & 4 Regimes \\
\hline \multicolumn{7}{|c|}{ Results for CEI } \\
\hline constant $\sigma$ & -28.6 & -12.3 & -9.3 & -22.4 & -11.7 & -12.8 \\
\hline reg.-dep. $\sigma$ & -25.4 & -58.8 & -13.2 & -19.8 & -20.0 & -8.0 \\
\hline \multicolumn{7}{|c|}{ Results for IP } \\
\hline constant $\sigma$ & -130.1 & -112.1 & -123.0 & -123.5 & -113.7 & -123.7 \\
\hline reg.-dep. $\sigma$ & -117.1 & -122.1 & -127.2 & -117.9 & -132.8 & -125.2 \\
\hline \multicolumn{7}{|c|}{ Results for LEI } \\
\hline constant $\sigma$ & -88.0 & -86.8 & -85.8 & -88.5 & -83.3 & -84.7 \\
\hline reg.-dep. $\sigma$ & -89.9 & -109.8 & -89.2 & -89.2 & -76.7 & -86.0 \\
\hline
\end{tabular}

Note: The table presents log predictive likelihood values for MS-AR models estimated for monthly growth rates of the Conference Board coincident economic index (CEI), industrial production index (IP), and the Conference Board leading economic index (LEI). The estimation sample used for obtaining a posterior sample of the model parameters covers the period January 1960 - December 2000. The remaining period January 2001 - October 2010 is used to compute log predictive likelihood values. Constant $\sigma$ stands for the model where in each subperiod (separated by a structural break in the variance) homoskedasticity is assumed. Regime-dependent (reg.-dep.) $\sigma$ stands for the model with regime-dependent heteroskedasticity together with a structural break in variances. $\mathrm{AR}(0)$ and $\mathrm{AR}(1)$ indicate models with no and firstorder autoregressive dynamics, respectively. Posterior results are based on 40,000 simulations of which the first 20,000 are discarded as burn-in sample. The convergence of the MCMC sampler is checked using statistical and visual inspection and in all model specifications convergence is assured.

Table 1 displays the log predictive likelihoods of the univariate models for CEI, IP and LEI, giving rise to several interesting observations. First, for the models with constant variances the predictive likelihood values tend to increase dramatically when increasing the number of regimes from two to three. This is most pronounced for CEI and IP. This trend continues when we move to a four-regime model for the CEI, albeit only slightly. For the LEI we also find an increase in predictive likelihood with the number of regimes, but this is much more modest than for the CEI and IP.

When the aim is to capture recessions and expansions in the most general sense with no further refinements, Markov-switching models with two regimes are the obvious candidates. In that case, it seems that allowing for regime-dependent heteroskedasticity is beneficial, in the sense that for the CEI and IP the predictive

\footnotetext{
${ }^{2}$ All remaining results are available upon request.
} 
likelihood is higher than for the models with constant variance. This does not hold, however, for the LEI. Interestingly, Table 1 shows that increasing the number of regimes in the model with regime-specific volatilities leads to a decline in predictive likelihood, which in many cases is quite substantial. In fact, for all three variables we find that three-regime models with constant variances provide superior predictive results than the two-regime models with regime-dependent heteroskedasticity.

When we compare the models with and without first-order autoregressive dynamics (denoted by $\mathrm{AR}(0)$ and $\mathrm{AR}(1)$ ), we observe mixed results. Especially when the number of regimes is restricted to only two, adding $\mathrm{AR}(1)$ dynamics provides an improvement in model predictions. The same, however, does not apply uniformly when more regimes are considered. It seems that regime-switching means (and variances) only are not sufficient to describe the cyclical behavior in these series completely in case only two regimes are allowed. In that case, the first-order autoregressive dynamics is useful to capture (part of) the remaining structure. With the increase in the number of regimes this limited effect vanishes completely, however. It is also important to note that unreported results indicate that the models with $\operatorname{AR}(1)$ dynamics lack robustness, in the sense that we find rather different parameter estimates for these models when using the initial estimation sample until December 2000 compared to using the complete sample period until October $2010 .^{3}$

The results show that for all variables the models with three and four regimes and with constant variances with a single structural break and no autoregressive dynamics provide relatively higher predictive likelihood values. ${ }^{4}$ Moreover, these models are robust against the sample chosen. Therefore, we display the results of

\footnotetext{
${ }^{3}$ Using the estimation sample until December 2000, the models with AR(1) dynamics give similar parameter estimates as the corresponding models without autoregressive dynamics. This is also the main source of the high predictive likelihood values for some of these models.

${ }^{4} \mathrm{~A}$ possible explanation for this finding may be that the regime-switching dynamics already provides sufficient flexibility to the model. Note that, a model with no AR terms but with Markov switching regime dynamics can be written as an ARMA specification, see Hamilton (1989) for details.
} 
these models in Tables 2, 3 and 4 for CEI, IP and LEI, respectively. We also include the results of the two regime model with regime-dependent heteroscedasticity and a single structural break for comparison. The posterior regime probabilities for these models are displayed in Figures 2, 3 and 4.

For the models with two regimes for CEI and IP, it seems that the variance dynamics is the driving force of the regimes rather than the mean dynamics. While the estimates for the mean growth rates are different in the two regimes, note that only for IP we find a negative growth rate for one of the regimes (but with a large posterior standard deviation), while for the CEI we find one regime with positive growth and one with essentially zero growth, on average. The differences in variances across regimes are much larger, in the sense that for CEI and IP the variances in the regimes with the highest mean growth are, respectively, four and six times smaller than the variances during the other regime. From Figures 2 and 3 we observe that the correspondence of the "low growth, high volatility" regime with the NBER recessions is far from perfect. The mild recessions of 1990-1 and 2001 are missed almost completely, while at other times temporary high volatility wrongfully signals a 'recession'; for example in 1987, 1996 and 2005 for the CEI. From the first panel of Figure 4, we see that the two regime model for the LEI can provide more accurate signals for recessions. Obviously, the mismatch between the NBER recession dates is not because of the low quality of signals but because of the leading property of this indicator. Still, it seems that at the onset of LEI's recessions there are mixed signals that are far from 0 or 1 especially for the period around 2006 .

The most interesting finding for the three regime models is that, in contrast to the existing evidence, the additional third regime is a recession type of regime rather than a recovery phase as in Sichel (1994) and Boldin (1996), among others. For all three variables the estimation results indicate the presence of separate phases of mild and severe recessions. For the CEI, the mean growth rate during mild recessions 
Table 2: Posterior results of selected univariate Markov-switching models for the CEI

\begin{tabular}{lccc} 
MS(2)AR(1) with regime-dependent $\sigma$ & & \\
Mean, $\mu_{j}$ & & Regime 1 & Regime 2 \\
Variance after the structural break, $\sigma_{j}^{2}$ & $0.22(0.02)$ & $0.01(0.13)$ \\
Most likely break date, $\tau$ & $0.04(0.01)$ & $0.16(0.05)$ \\
Ratio between st.dev's, $\delta$ & \multicolumn{2}{c}{$1984-1$} \\
Autoregressive coefficient, $\phi$ & \multicolumn{2}{c}{$1.51(0.15)$} \\
& & $0.37(0.08)$ \\
Transition & Regime 1 & $0.93(0.04)$ & $0.07(0.04)$ \\
probabilities, $p_{i j}$ & Regime 2 & $0.25(0.09)$ & $0.75(0.09)$
\end{tabular}

$\operatorname{MS}(3) \operatorname{AR}(0)$ with constant $\sigma$

Mean, $\mu_{j}$

Variance after the structural break, $\sigma^{2}$

Most likely break date, $\tau$

Ratio between st.dev's, $\delta$

$\begin{array}{ccc}\text { Regime 1 } & \text { Regime 2 } & \text { Regime 3 } \\ 0.35(0.03) & 0.10(0.04) & -0.45(0.06) \\ 0.04(0.00) & \\ 1984-2 & \\ 1.54(0.10) & \\ \text { Regime 1 } & \text { Regime 2 } & \text { Regime 3 } \\ 0.96(0.02) & 0.04(0.01) & 0.01(0.01) \\ 0.03(0.02) & 0.92(0.03) & 0.05(0.02) \\ 0.14(0.06) & 0.11(0.06) & 0.75(0.07)\end{array}$

$\operatorname{MS}(4) \operatorname{AR}(0)$ with constant $\sigma$

Mean, $\mu_{j}$

Variance after the structural break, $\sigma^{2}$

Most likely break date, $\tau$

Ratio between st.dev's, $\delta$

\begin{tabular}{cccc} 
Regime 1 & Regime 2 & Regime 3 & Regime 4 \\
$0.67(0.20)$ & $0.25(0.07)$ & $-0.00(0.02)$ & $-0.52(0.06)$ \\
$0.04(0.00)$ & & \\
$1984-2$ & & \\
$1.48(0.14)$ & & \\
\multicolumn{2}{r}{} & & \\
Regime 1 & Regime 2 & Regime 3 & Regime 4 \\
$0.53(0.28)$ & $0.31(0.20)$ & $0.07(0.09)$ & $0.09(0.09)$ \\
$0.03(0.03)$ & $0.92(0.05)$ & $0.04(0.03)$ & $0.01(0.02)$ \\
$0.03(0.07)$ & $0.08(0.10)$ & $0.81(0.17)$ & $0.08(0.06)$ \\
$0.10(0.06)$ & $0.11(0.07)$ & $0.13(0.09)$ & $0.66(0.10)$
\end{tabular}

Note: The table presents posterior means and standard deviations (in parentheses) of parameters in the selected MS-AR models estimated for monthly growth rates of the Conference Board coincident economic index (CEI) over the period January 1960 - October 2010. MS $(J) \operatorname{AR}(K)$ is the abbreviation for the Markov-switching model with $J$ regimes and with $K^{\text {th }}$ order autoregressive dynamics. Constant $\sigma$ stands for the model where in each subperiod (separated by a structural break in the variance) homoskedasticity is assumed. Regime-dependent $\sigma$ stands for the model with regime-dependent heteroskedasticity together with a structural break in variances. The most likely break date is defined as the mode of the posterior distribution of $\tau$. Posterior results are based on 40,000 simulations of which the first 20,000 are discarded as burn-in sample. The convergence of the MCMC sampler is checked using statistical and visual inspection and in all model specifications convergence is assured. 
Table 3: Posterior results of selected univariate Markov-switching models for the IP

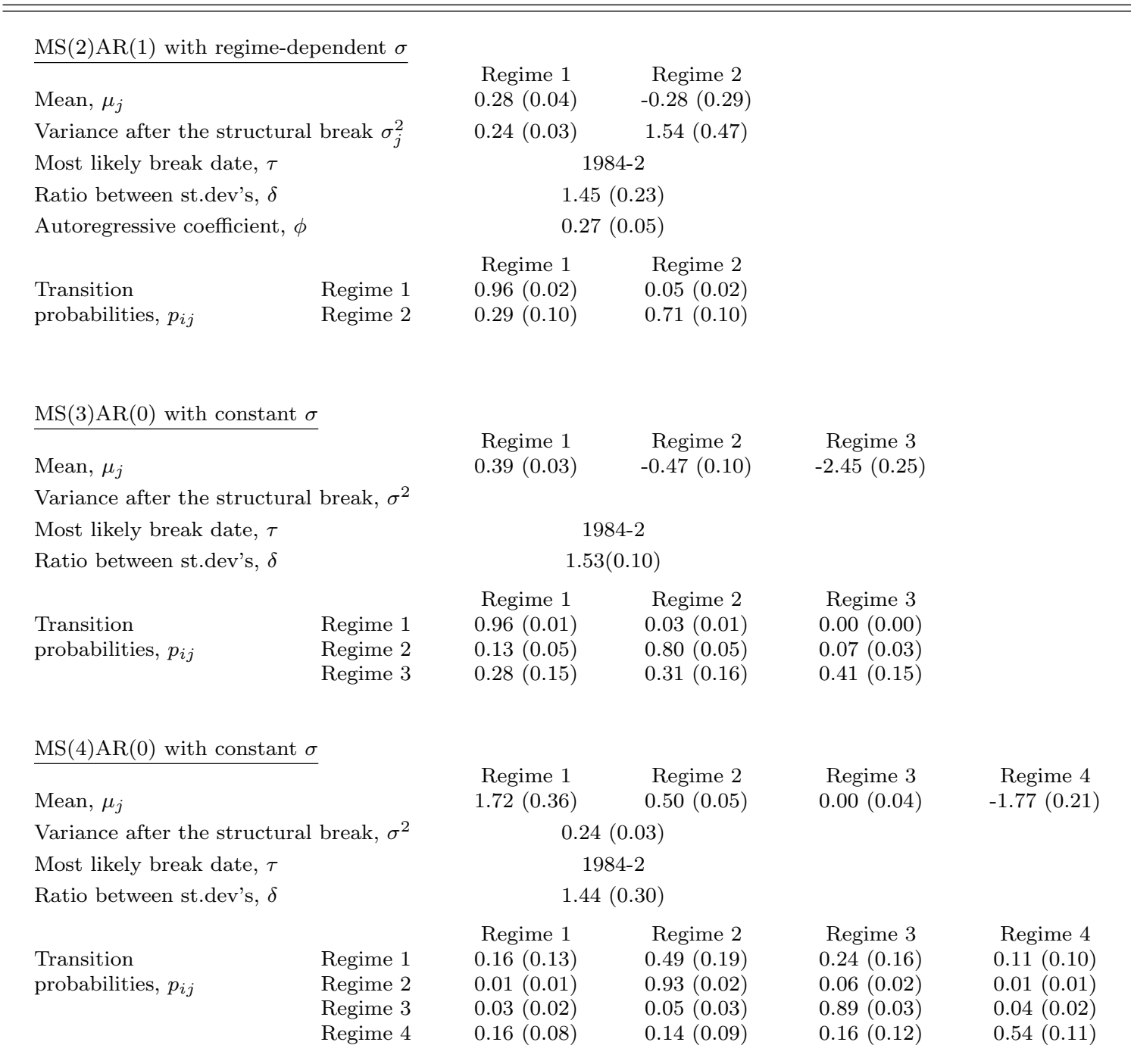

Note: The table presents posterior means and standard deviations (in parentheses) of parameters in the selected MS-AR models estimated for monthly growth rates of industrial production (IP) over the period January 1960 October 2010. See Table 2 for further details. 
Table 4: Posterior results of selected univariate Markov-switching models for the LEI

\begin{tabular}{|c|c|c|c|c|c|}
\hline \multicolumn{6}{|c|}{$\operatorname{MS}(2) \operatorname{AR}(1)$ with regime-dependent $\sigma$} \\
\hline \multicolumn{2}{|c|}{ Mean, $\mu_{j}$} & $\begin{array}{c}\text { Regime } 1 \\
0.41(0.06)\end{array}$ & $\begin{array}{c}\text { Regime } 2 \\
-0.33(0.17)\end{array}$ & & \\
\hline \multicolumn{2}{|c|}{ Variance after the structural break $\sigma_{j}^{2}$} & $0.20(0.03)$ & $0.40(0.17)$ & & \\
\hline \multicolumn{2}{|c|}{ Most likely break date, $\tau$} & \multicolumn{2}{|c|}{$1984-2$} & & \\
\hline \multicolumn{2}{|c|}{ Ratio of st.dev's, $\delta$} & \multicolumn{2}{|c|}{$1.31(0.12)$} & & \\
\hline \multicolumn{2}{|c|}{ Autoregressive coefficient, $\phi$} & \multicolumn{2}{|c|}{$0.26(0.07)$} & & \\
\hline $\begin{array}{l}\text { Transition } \\
\text { probabilities, } p_{i j}\end{array}$ & $\begin{array}{l}\text { Regime } 1 \\
\text { Regime } 2\end{array}$ & $\begin{array}{c}\text { Regime 1 } \\
0.97(0.01) \\
0.08(0.03)\end{array}$ & $\begin{array}{c}\text { Regime } 2 \\
0.03(0.01) \\
0.92(0.03)\end{array}$ & & \\
\hline \multicolumn{6}{|c|}{$\operatorname{MS}(3) \operatorname{AR}(0)$ with constant $\sigma$} \\
\hline \multicolumn{2}{|l|}{ Mean, $\mu_{j}$} & $\begin{array}{c}\text { Regime 1 } \\
0.46(0.03)\end{array}$ & $\begin{array}{c}\text { Regime } 2 \\
-0.27(0.07)\end{array}$ & $\begin{array}{c}\text { Regime } 3 \\
-1.64(0.30)\end{array}$ & \\
\hline \multicolumn{2}{|c|}{ Variance after the structural break, $\sigma^{2}$} & \multicolumn{2}{|c|}{$0.20(0.02)$} & & \\
\hline \multicolumn{2}{|c|}{ Most likely break date, $\tau$} & \multicolumn{2}{|c|}{ 1983-7 } & & \\
\hline \multicolumn{2}{|c|}{ Ratio between st.dev's, $\delta$} & \multicolumn{2}{|c|}{$1.37(0.09)$} & & \\
\hline $\begin{array}{l}\text { Transition } \\
\text { probabilities, } p_{i j}\end{array}$ & $\begin{array}{l}\text { Regime } 1 \\
\text { Regime } 2 \\
\text { Regime } 3\end{array}$ & $\begin{array}{c}\text { Regime } 1 \\
0.96(0.01) \\
0.07(0.03) \\
0.23(0.12)\end{array}$ & $\begin{array}{c}\text { Regime } 2 \\
0.03(0.01) \\
0.90(0.03) \\
0.11(0.10)\end{array}$ & $\begin{array}{c}\text { Regime } 3 \\
0.00(0.00) \\
0.03(0.02) \\
0.66(0.13)\end{array}$ & \\
\hline \multicolumn{6}{|c|}{$\operatorname{MS}(4) \operatorname{AR}(0)$ with constant $\sigma$} \\
\hline \multicolumn{2}{|l|}{ Mean, $\mu_{j}$} & $\begin{array}{c}\text { Regime 1 } \\
1.06(0.11)\end{array}$ & $\begin{array}{c}\text { Regime } 2 \\
0.40(0.04)\end{array}$ & $\begin{array}{c}\text { Regime } 3 \\
0.00(0.04)\end{array}$ & $\begin{array}{c}\text { Regime } 4 \\
-0.98(0.14)\end{array}$ \\
\hline \multicolumn{2}{|c|}{ Variance after the structural break, $\sigma^{2}$} & \multicolumn{2}{|c|}{$0.17(0.02)$} & & \\
\hline \multicolumn{2}{|c|}{ Most likely break date, $\tau$} & \multicolumn{2}{|c|}{ 1984-2 } & & \\
\hline \multicolumn{2}{|c|}{ Ratio between st.dev's, $\delta$} & \multicolumn{2}{|c|}{$1.31(0.12)$} & & \\
\hline $\begin{array}{l}\text { Transition } \\
\text { probabilities, } p_{i j}\end{array}$ & $\begin{array}{l}\text { Regime } 1 \\
\text { Regime } 2 \\
\text { Regime } 3 \\
\text { Regime } 4\end{array}$ & $\begin{array}{c}\text { Regime } 1 \\
0.71(0.08) \\
0.01(0.01) \\
0.05(0.02) \\
0.07(0.04)\end{array}$ & $\begin{array}{c}\text { Regime } 2 \\
0.18(0.07) \\
0.95(0.02) \\
0.02(0.02) \\
0.03(0.03)\end{array}$ & $\begin{array}{c}\text { Regime } 3 \\
0.04(0.03) \\
0.03(0.02) \\
0.83(0.05) \\
0.22(0.10)\end{array}$ & $\begin{array}{c}\text { Regime } 4 \\
0.03(0.02) \\
0.01(0.01) \\
0.10(0.05) \\
0.68(0.10)\end{array}$ \\
\hline
\end{tabular}

Note: The table presents posterior means and standard deviations (in parentheses) of parameters in the selected MS-AR models estimated for monthly growth rates of the Conference Board leading economic index (LEI) over the period January 1960 - October 2010. See Table 2 for further details. 
Figure 2: Posterior regime probabilities for selected models of the CEI
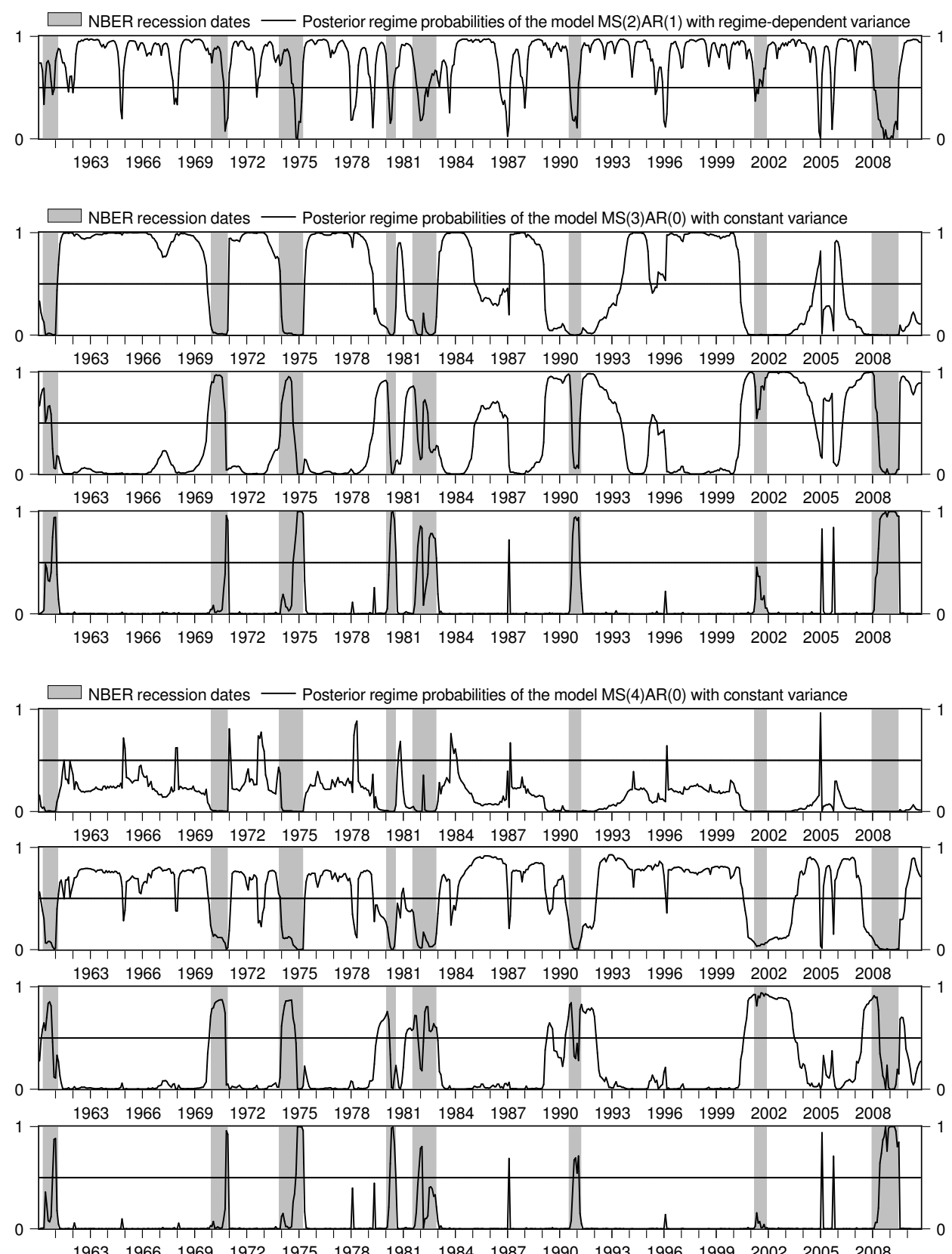

$\begin{array}{llllllllllllllll}1963 & 1966 & 1969 & 1972 & 1975 & 1978 & 1981 & 1984 & 1987 & 1990 & 1993 & 1996 & 1999 & 2002 & 2005 & 2008\end{array}$

Note: The solid lines are the posterior regime probabilities for the selected models summarized in Table 2 for the Conference Board coincident economic index (CEI). The shaded areas indicate the US recessions as determined by the NBER Business Cycle Dating Committee. 
Figure 3: Posterior regime probabilities for selected models of IP
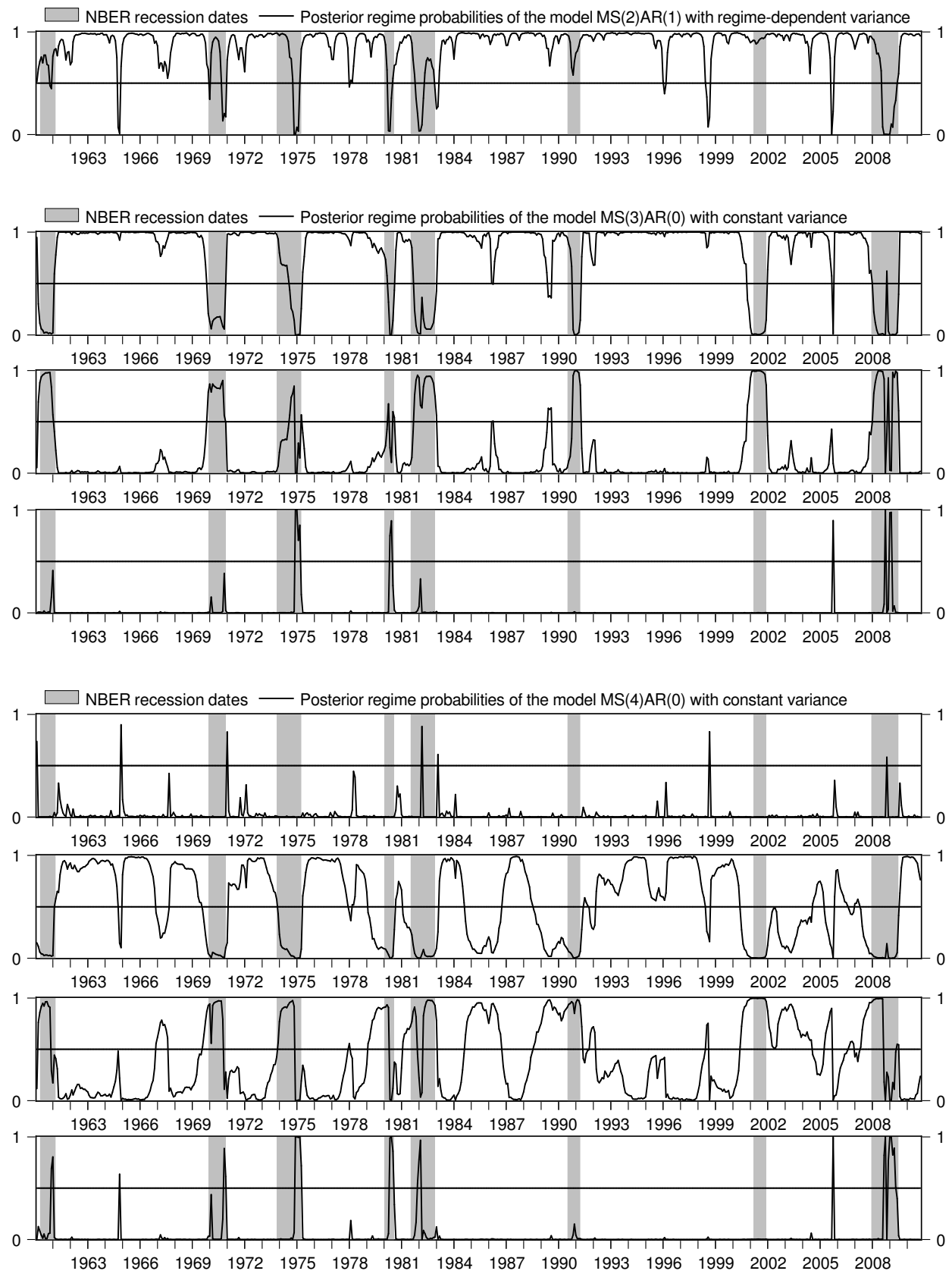

Note: The solid lines are the posterior regime probabilities for the selected models summarized in Table 3 for industrial production (IP). The shaded areas indicate the US recessions as determined by the NBER Business Cycle Dating Committee. 
Figure 4: Posterior regime probabilities for selected models of LEI
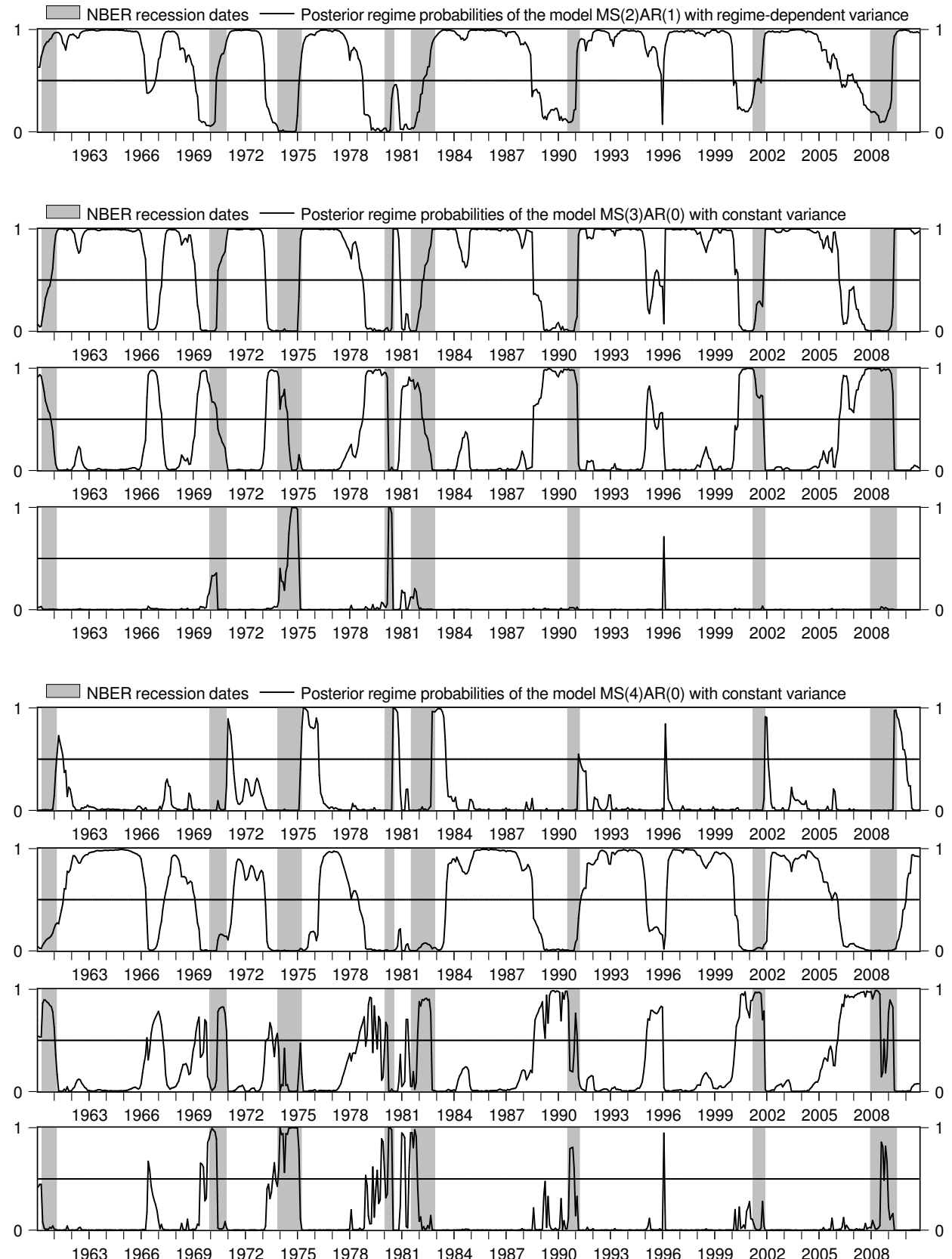

Note: The solid lines are the posterior regime probabilities for the selected models summarized in Table 4 for the Conference Board leading economic index (LEI). The shaded areas indicate the US recessions as determined by the NBER Business Cycle Dating Committee. 
is positive but rather close to zero, while severe recessions are characterized by a large negative mean growth rate of -0.45 percent per month. One drawback of this specification, however, is that it produces false signals following the recessions of 1990-1 and 2001, in the sense that the probability of the second regime is fairly high for a considerable period of time after the end of these recessions. Unreported results indicate very similar results for ENP; hence, the second regime also captures those periods with basically no growth or limited growth in employment conditions, in particular the jobless recovery periods that are not consistent with the NBER recession dating chronology.

The three regime model of IP on the other hand signals very accurate predictions of the recessions consistent with NBER peak and trough dates. As shown by the graphs in the middle panel of Figure 3, the third regime captures the latter part of the recessions in 1974, 1980 and 2008. At -2.45 percent the mean growth rate in this regime is much lower than that of the second regime, representing a mild recession phase with mean growth of -0.47 percent. Clearly, the additional third regime does not capture isolated outlying observations as the predictive likelihoods dramatically increase with the inclusion of this regime and also the probability of staying in this regime is far from 0 .

The same conclusion applies to the LEI. The third regime has similar characteristics as that for IP with a very large mean contraction rate, but with phase shifts in terms of the timing of the start of this regime (as expected). It is also important to note that inclusion of the third regime actually improves the recession signals of the LEI compared to the two regime model, especially around 2006.

Unreported results for the other variables lead to similar conclusions. The main exception is that for MTS and PI the additional third regime indicates a recovery phase following recessions. Besides the fact that these models have very low 
predictive likelihoods, they also seriously lack robustness. ${ }^{5}$

Given the distinction between mild and severe recessions in the three regime models and previous evidence for the existence of a recovery phase, one might expect superior performance of four regime models, as these may be able to capture both phenomena simultaneously. However, this turns out not to be the case. The estimation results in Tables 2-4 and Figures 2-4 indicate that the additional fourth regime does not represent a recovery regime, except for the LEI to some extent. In particular for IP, the regime with the highest mean growth rate (1.72) captures only a few outliers, as shown by the occasional spikes in regime probability in Figure 3 and the low probability (0.16) of staying in the first regime. Albeit less dramatically, the same applies to the CEI. These results are in line with the predictive likelihoods in Table 1 where we find slight improvements for the four regime model (compared to the three regime model) for the LEI and the CEI, but a worsening for IP.

When the break dates of the variances are considered, there is a consensus across all the variables and across the various model specifications. The estimates indicate the first quarter of 1984 as the most likely break date, in line with existing evidence, see McConnell and Perez-Quiros (2000) and Sensier and van Dijk (2004), among others. The ratios of the standard deviations before and after the break are approximately 1.5 for the CEI and IP and 1.35 for the LEI, confirming the substantial reduction in volatility in macroeconomic fluctuations due to the Great Moderation.

The results from the univariate analysis indicate that the Markov-switching models with three regimes provide a much improved description of the business cycle dynamics compared to the models with two regimes only. While the models for the CEI show a dramatic increase in predictive likelihoods as the number of regimes is increased, this does not translate into an improved recession signal. We attribute this to the aggregation of different variables with distinct business cycle features to

\footnotetext{
${ }^{5}$ Detailed results are available upon request.
} 
construct the CEI, in particular ENP showing slow ('jobless') recovery, IP showing clear signs of the occurrence of 'severe recessions', and PI and MTS showing quick recoveries following contractions. On the other hand, IP and LEI provide first, more accurate predictions brought about by the additional third regime (as indicated by the predictive likelihoods); second, robust models with estimates that are not sensitive to the sample chosen; and third, clear signals of the recession dates and recession types (see Figures 3 and 4). We therefore proceed in the next section with a bivariate analysis of IP and LEI using an MS-VAR model.

\section{Multivariate analysis}

The posterior regime probabilities of the univariate MS models for IP and LEI indicate that both variables display similar characteristics and dynamics in their business cycle regimes, see Figures 3 and 4. We therefore consider the joint modeling of these two series, to obtain a clearer picture of the US business cycle. Obviously, there is a phase shift in the timing of the regime changes for the two variables, as IP is a coincident variable while the LEI leads the business cycle. Hence, when constructing a bivariate MS-VAR model for IP and LEI this phase shift has to be taken into account. Moreover, the time shift seems to be asymmetric across the business cycle. Indeed, using a two-regime MS-VAR model Paap et al. (2009) show that the LEI leads business cycle peaks by twelve months and troughs by three months, on average. In this paper we extend the analysis of Paap et al. (2009) to more than two regimes using an adjusted version of the model proposed by Cakmakli et al. (2011).

The model of Cakmakli et al. (2011) is a bivariate MS-VAR model with 'imperfect synchronization' of the cycles in the two variables due to asymmetric phase shifts of a single underlying Markov regime-switching process. The key feature of this model is that it allows for any number of regimes $J \geq 2$ and that the amount of 
the phase shifts can be different across regimes. Furthermore, regime-dependent heteroskedasticity can easily be implemented.

Let $y_{1, t}$ and $y_{2, t}$ denote the observations of the IP and LEI growth rates in month $t$ for $t=1, \ldots, T$. As in the univariate case, we assume that the $J$ phases of the business cycle are characterized by different means of $y_{1, t}$ and $y_{2, t}$. We assume that autoregressive coefficients are constant across regimes. In case of first-order autoregressive dynamics this leads to the model specification

$$
\begin{aligned}
& y_{1, t}-\mu_{1, S_{1, t}}=\phi_{1,1}\left(y_{1, t-1}-\mu_{1, S_{1, t-1}}\right)+\phi_{1,2}\left(y_{2, t-1}-\mu_{2, S_{2, t-1}}\right)+\varepsilon_{1, t}, \\
& y_{2, t}-\mu_{2, S_{2, t}}=\phi_{2,1}\left(y_{1, t-1}-\mu_{1, S_{1, t-1}}\right)+\phi_{2,2}\left(y_{2, t-1}-\mu_{2, S_{2, t-1}}\right)+\varepsilon_{2, t},
\end{aligned}
$$

where $S_{l, t}$ are latent multinomial variables taking the value $j$ if $y_{l, t}$ is in regime $j$ at time $t$, and where $\mu_{l, S_{l, t}}=\mathrm{E}\left[y_{l, t} \mid S_{l, t}\right]$ denotes the unconditional mean of $y_{l, t}$ in regime $S_{l, t}$ for $l=1,2$. The disturbances are assumed to be normally distributed with mean zero and time-varying covariance matrix $\Sigma_{t}$, that is,

$$
\left(\varepsilon_{1, t}, \varepsilon_{2, t}\right)^{\prime} \sim N I D\left(0, \Sigma_{t}\right)
$$

Before we discuss the specification of the covariance matrix $\Sigma_{t}$, we first consider the properties of the regime indicators $S_{l, t}$.

To model the dynamics in the regime indicators, we use again a first-order homogenous Markov process. A natural and elegant approach to model the systematic phase shifts between the cycles of LEI and IP is to assume that the cycle in $y_{2, t}$ leads the cycle in $y_{1, t}$ by $\kappa$ periods (Hamilton and Perez-Quiros, 1996)

$$
S_{2, t-\kappa}=S_{1, t}
$$

In other words, there is a common cycle but it affects the different variables with a certain phase shift. We refer to this case as imperfect synchronization with 'symmet- 
ric' phase shifts (SPS), where symmetry refers to the fact that all possible regime transitions in the two variables differ by the same number of time periods $\kappa$.

The specification in (7) may still be too restrictive, in the sense that the phase shift of the cycle may well be different for different regimes transitions. For example, leading indicator variables typically have a considerably longer lead time at business cycle peaks than at troughs, see The Conference Board (2001). For this purpose, Paap et al. (2009) consider a two-regime model with possibly different phase shifts $\kappa_{1}$ and $\kappa_{2}$ for these two types of turning points. Cakmakli et al. (2011) generalize the idea of imperfect synchronization with 'asymmetric' phase shifts (APS) to multiple regimes $(J>2)$ by specifying

$$
S_{2, t-\kappa_{S_{1, t}}}=S_{1, t}
$$

where the subscript $S_{1, t}$ to $\kappa$ indicates that the regime indicator is shifted by a possibly different number of time periods $\kappa_{j}$ for each regime $j=1, \ldots, J$. Put differently, we assume that the lead time is different per regime, such that each regime in the LEI starts earlier by $\kappa_{j}$ periods than the corresponding regimes in the IP, see Cakmakli et al. (2011) for model details. Note that the specification in (8) embeds imperfect synchronization with symmetric phase shifts (all $\kappa_{j} \neq 0$ but equal) as a special case, as well as what we might call perfect synchronization (all $\left.\kappa_{j}=0\right){ }^{6}$

To finalize the model specification, we return to the specification of the covariance matrix $\Sigma_{t}$ in (6). As in the univariate case, we consider constant and regimedependent variances, while in both cases we allow for a single structural break in volatilities to accommodate the Great Moderation. To facilitate the specification,

\footnotetext{
${ }^{6}$ The specification in (8) is not complete, in the sense that it may lead to situations where for some time periods $S_{2, t}$ is assigned to multiple regimes (or to no regime at all). We impose the rule that the regime with the larger phase shift parameter determines the regime of $S_{2, t}$ in such conflicting (or 'empty') periods, see Cakmakli et al. (2011) for discussion.
} 
the covariance matrix is decomposed into variances and correlations, as

$$
\Sigma_{t}=D_{t} R D_{t}
$$

where $D_{t}=\operatorname{diag}\left(\sigma_{1, t}, \sigma_{2, t}\right)$ is a diagonal matrix with the standard deviations of the error terms as diagonal elements and $R$ is a matrix with ones on the diagonal and the correlation $\rho$ as the off-diagonal element (see, for example, Barnard et al., 2000). In case of regime-dependent variances, the elements of $D_{t}$ are modeled as

$$
\sigma_{l, t}=\left\{\begin{array}{ll}
\delta \sigma_{l, S_{l, t}} & \text { if } t<\tau \\
\sigma_{l, S_{l, t}} & \text { if } t \geq \tau
\end{array} \quad \text { for } l=1,2\right.
$$

where we allow for a single break in the variances at time $\tau$ captured by the single scaling factor $\delta$. To keep the complexity of the model at a feasible level, the correlation parameter $\rho$ is assumed to be regime-independent and constant over time unlike the variances. Assuming constant variances across regimes boils down to imposing $\sigma_{l, S_{l, t}}=\sigma_{l}$. In this case, we also allow the correlation to change at the time of the structural break in the volatilities.

\subsection{Bayesian Inference}

As in the univariate case, we opt for a Bayesian approach to estimate the parameters of the bivariate MS-VAR model with imperfect synchronization. Note that this greatly facilitates inference on the value of the discrete lead time parameters $\kappa_{j}, j=$ $1, \ldots, J$ (and the timing of the structural break in variances $\tau$ ), which is problematic in a frequentist analysis.

As we want our posterior results to be driven by the data rather than the prior distributions, we impose rather diffuse prior specifications for the model parameters. Details of the adopted priors are provided in Appendix A.1. The posterior 
distribution of the model parameters and unobserved states is proportional to the product of the complete likelihood function and the joint prior, which is the product of the marginal prior distributions. The complete likelihood function is derived in Appendix A.2. As in the univariate case, we use Gibbs sampling together with data augmentation to obtain posterior results. Details on the derivation of the conditional posterior distributions are given in Appendix A.3. Model selection is carried out in a similar manner as in the univariate analysis. We compute marginal likelihood values to compare models with different types of synchronization.

\subsection{Empirical Results}

Following the evidence from our univariate analysis we estimate MS-VAR models with two or three regimes. For the relation between the regime dynamics in IP and LEI, we consider both possibilities discussed in the previous section, namely (i) imperfect synchronization with a single phase shift of the common cycle (SPS); and (ii) imperfect synchronization with asymmetric (or regime-specific) phase shifts (APS).

For the models with imperfect synchronization, we specify the set of admissible phase shift parameters to be in the interval $[-15,15]$. Hence any regime in the cycle of the LEI can lead/lag the corresponding regime in the IP by at most 15 months. We also restrict the differences between the phase shift parameters for different regimes to be one year at most. For the autocorrelation dynamics, we impose $\phi_{1,1}=\phi_{2,1}=0$ as marginal likelihoods computed using mildly informative priors for these parameters are hardly affected by this restriction, see Paap et al. (2009) and Hamilton and Perez-Quiros (1996) for a similar result. The prior for the break date parameter $\tau$ is set in such a way that the break in the variances can occur everywhere apart from the first and last 6 months of the sample.

We use the sample from January 1960 until December 2000 for estimation of the 
model and the remaining period from January 2001 until October 2010 to compute the predictive likelihood as in the univariate analysis. We focus on the predictive ability of the models for the IP series, as compared to LEI prediction of IP is of first-order importance.

The marginal and predictive likelihood results in Table 5 show three interesting features. First, both the marginal and the predictive likelihood increase when moving from the SPS to the APS specification, with the only exception of the three regime model with regime-dependent variances. This clearly shows the importance of allowing for different phase shifts for the different regimes. Second, in line with our previous results inclusion of the third regime increases the predictive likelihood values in most cases. Third, imposing equal variances across regimes improves the predictive likelihood for all specifications. ${ }^{7}$ Combining these three findings, we conclude that the three regime model with asymmetric phase shifts and equal variances across regimes performs best in terms of predictive ability. The parameter estimates (posterior means and standard deviations) for this model specification are displayed in Table 6 and posterior probabilities of regimes are shown in Figure 5.

The estimation results in Table 6 indicate once again the necessity of distinguishing between two different types of economic declines. ${ }^{8}$ On the one hand, the regime with mean growth rates of -0.5 and -0.25 for IP and the LEI can be labeled as 'mild recessions'. On the other hand, the regime with much larger declines of -2.4 and -1.5 percent could be called 'severe recessions'.

The posterior regime probabilities in Figure 5 indicate that severe recessions occurred in 1974-5, 1980, and 2008-9, with all three being preceded by a period of

\footnotetext{
${ }^{7}$ This observation is not valid for marginal likelihoods, however, marginal likelihoods of the models with constant and regime-dependent variances are not comparable as we specify improper prior distributions for the variance parameters.

${ }^{8}$ Note that the estimation results also show that it is worthwhile to combine the information in IP and the LEI by jointly modeling these two series. The precision of the parameter estimates is improved compared to the univariate models, as well as the accuracy of the posterior regime probabilities. Finally, the predictive likelihood values obtained from the bivariate model are also higher than those from the corresponding univariate three regime model for IP.
} 
Table 5: Comparison of multivariate MS-VAR models estimated for IP and LEI

\begin{tabular}{cccccc}
\hline \hline & \multicolumn{2}{c}{ Log marginal likelihood } & & \multicolumn{2}{c}{ Log predictive likelihood } \\
\cline { 2 - 3 } \cline { 5 - 6 } constant $\Sigma$ & reg.-dep. $\Sigma$ & & constant $\Sigma$ & reg.-dep. $\Sigma$ \\
\hline \multirow{2}{*}{ SPS } & -1196.4 & -1144.8 & & -132.2 & -152.0 \\
APS & -1164.7 & -1120.5 & & -124.8 & -148.2 \\
& & & 3 regimes & \\
SPS & -1228.8 & -1150.0 & & -116.3 & -144.1 \\
APS & -1136.6 & -1117.6 & & -107.9 & -144.4 \\
\hline
\end{tabular}

Note: The table presents log marginal likelihoods and log predictive likelihoods of the competing MS-VAR models with (i) two or three regimes; (ii) imperfect synchronization with a single phase shift (SPS) or regime-specific (or asymmetric) phase shifts (APS) of the common cycle and (iii) homoskedastic error terms (constant $\Sigma$ ) or with regime-dependent variances (regime-dependent $\Sigma$ ), where in both cases the variances are subject to a single structural break and the correlation is assumed to be the same across regimes and constant over time. The models are applied to monthly growth rates of industrial production (IP) and the Conference Board leading economic index (LEI) for the sample period January 1960 - October 2010. Marginal likelihoods are those obtained when the models are estimated for the complete sample period. Predictive likelihood values are for the period January 2001 - October 2010, which are computed conditional on the posterior distributions of the model parameters obtained with an estimation sample ending in December 2000. Posterior results for computing log marginal likelihoods and predictive likelihoods are based on 40,000 simulations of which the first 20,000 are discarded as burn-in sample. The convergence of the MCMC sampler is checked using statistical and visual inspection and in all model specifications convergence is assured. 
Table 6: Posterior results for three regime MS-VAR model for IP and LEI with asymmetric synchronization, homoskedastic shocks and a structural break in the variances

\begin{tabular}{|c|c|c|c|c|}
\hline Growth Rates, $\mu_{l, j}$ & $\begin{array}{l}\text { Regime } 1 \\
\text { Regime } 2 \\
\text { Regime } 3\end{array}$ & $\begin{array}{c}\text { IP } \\
0.371(0.028) \\
-0.535(0.087) \\
-2.395(0.200)\end{array}$ & $\begin{array}{c}\text { LEI } \\
0.407(0.033) \\
-0.242(0.060) \\
-1.508(0.219)\end{array}$ & \\
\hline Most likely break date, $\tau$ & $1984-2$ & & & \\
\hline $\begin{array}{l}\text { Variance before break } \\
\text { Variance after break }\end{array}$ & & $\begin{array}{l}0.526(0.049) \\
0.245(0.022)\end{array}$ & $\begin{array}{l}0.388(0.035) \\
0.221(0.019)\end{array}$ & \\
\hline $\begin{array}{l}\text { Correlation between the } \\
\text { error terms of IP and LEI before break } \\
\text { after break }\end{array}$ & & \multicolumn{2}{|c|}{$\begin{array}{l}0.457(0.052) \\
0.263(0.057)\end{array}$} & \\
\hline $\begin{array}{l}\text { Autoregressive } \\
\text { dynamics }\end{array}$ & & $\begin{array}{c}\phi_{1,2} \\
0.059(0.050)\end{array}$ & $\begin{array}{c}\phi_{2,2} \\
0.162(0.043)\end{array}$ & \\
\hline $\begin{array}{l}\text { Transition } \\
\text { Probabilities }\end{array}$ & $\begin{array}{l}\text { Regime } 1 \\
\text { Regime } 2 \\
\text { Regime } 3\end{array}$ & $\begin{array}{c}\text { Regime } 1 \\
0.973(0.008) \\
0.112(0.041) \\
0.272(0.119)\end{array}$ & $\begin{array}{c}\text { Regime 2 } \\
0.025(0.008) \\
0.831(0.050) \\
0.169(0.105)\end{array}$ & $\begin{array}{l}\text { Regime } 3 \\
0.002(0.002) \\
0.057(0.027) \\
0.559(0.130)\end{array}$ \\
\hline Lead/Lag times & & $\begin{array}{c}\text { Regime } 1 \\
2.042(0.201)\end{array}$ & $\begin{array}{c}\text { Regime } 2 \\
10.838(1.326)\end{array}$ & $\begin{array}{c}\text { Regime } 3 \\
6.231(1.722)\end{array}$ \\
\hline
\end{tabular}

Note: The table presents posterior means and standard deviations (in parentheses) of parameters of the MS-VAR model with three regimes and a single structural change in the covariance matrix, estimated using monthly growth rates of IP and LEI over the period January 1960 - October 2010. The most likely break date is defined as the mode of the posterior distribution of $\tau$. Posterior results are based on 40,000 draws from the parameter distribution and convergence is verified using visual and statistical tools. Number of burn-in simulations is 20,000 . The convergence of the MCMC sampler is checked using statistical and visual inspection and in all model specifications convergence is assured. 
mild recession. We return to this issue in detail in the next section. Interestingly, the first two severe recessions were followed immediately by expansion periods, while the most recent one was followed by another mild recession period before entering the expansion regime in June 2009. Note that the LEI did signal the severe recessions in 1974-5 and 1980 in advance, but it failed to provide an early warning for the most recent one in 2008-9. This is due to the fact that this phase was followed by a mild recession period again, unlike the previous crises in 1974-5 and 1980. This indicates the necessity for more sophisticated leading indicators or at least multiple indicators each of which pre-signaling specific phases of the state of the economy as earlier as possible.

The regime probabilities in 2008-9 show a peculiar pattern anyway. As seen in Figure 5, the posterior probabilities convincingly indicate a severe recession during the period September 2008 - January 2009, but interrupted by a spike in the probability of the expansion regime in October 2008 (and in the probability of the mild recession regime in November and December). This corresponds with a $1.26 \%$ increase in IP during that month, following the large decline of 4.04\% in September. One possible explanation for this event might be the very low base level for the October growth rate. A more theoretical explanation is based on Romer (1990)'s uncertainty hypothesis, that is, increasing stock market volatility gives rise to consumers deferring their consumption on durable goods and increasing their consumption on nondurables. Indeed, calculating monthly realized stock market volatility based on daily S\&P 500 returns we find that volatility was at its highest level in October and November 2008 (the second highest level during the entire postwar period after the October 1987 crash). Interestingly, durable goods IP data in these months showed a $2.3 \%$ decline, whereas nondurable goods increased by $2.7 \%$, the only positive realization between May 2008 and March 2009.

While showing much stronger declines in output, severe recessions last consid- 
Figure 5: Posterior probabilities of regimes of IP and LEI
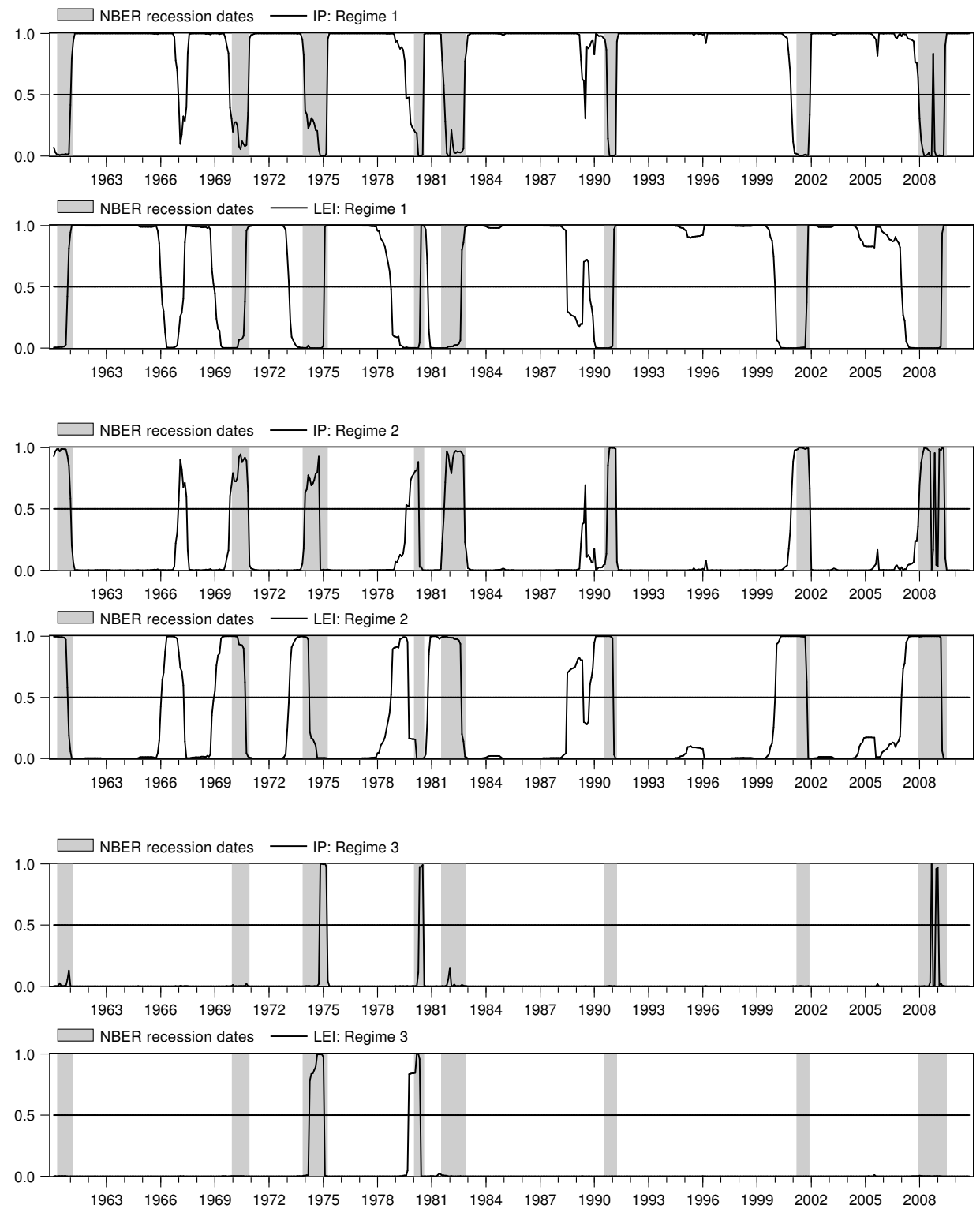

Note: The solid lines are the posterior regime probabilities for industrial production (IP) and the Conference Board leading economic index (LEI) for the three regime MS-VAR model with imperfect synchronization with asymmetric (or regime-specific) phase shifts and with a single structural break in the covariance matrix, summarized in Table 6. The shaded areas indicate the US recessions as determined by the NBER Business Cycle Dating Committee. 
erably shorter than mild contractions. According to the transition probability estimates the average duration of a typical mild recession is $1 /(1-0.83) \approx 6$ months, whereas severe recessions only last on average $1 /(1-0.56) \approx 2.2$ months.

The posterior distribution of the lead/lag parameters $\kappa_{j}, j=1, \ldots, 3$ is shown in Figure 6. For the expansion, mild recession and severe recession phases, the mean lead times are 2, 10.8 and 6.2 months, respectively. Posterior mode values point out a very similar picture with values of 2,12 and 7 months for the three regimes. As the posterior standard deviations are quite small and all the marginal distributions are unimodal, mode and mean values are very close to each other. These results demonstrate that the LEI is more successful in signaling upcoming (mild) recessions than expansions, in agreement with results in Paap et al. (2009) based on a tworegime model for the CEI and LEI. In addition, our results suggest that the LEI is somewhat less timely for severe recessions, although the lead time of just over half a year is still substantial.

Based on the estimates of the $\kappa_{j}$ parameters we can also draw inference about the relative duration of each regime. Recall that the estimation results show that all recession periods start as a mild downturn. Hence, based on the modes of the posterior distributions a typical expansion regime in the LEI is $(2-12=-10) 10$ months shorter than that of IP. Recessions can either remain mild for their entire duration, or worsen into a severe downturn. In the former case, a typical (mild) recession in the LEI is $(12-2=10) 10$ months longer than that of IP while in the latter case it is only (12-7=5) 5 months longer. 
Figure 6: Posterior distribution of lead time parameters
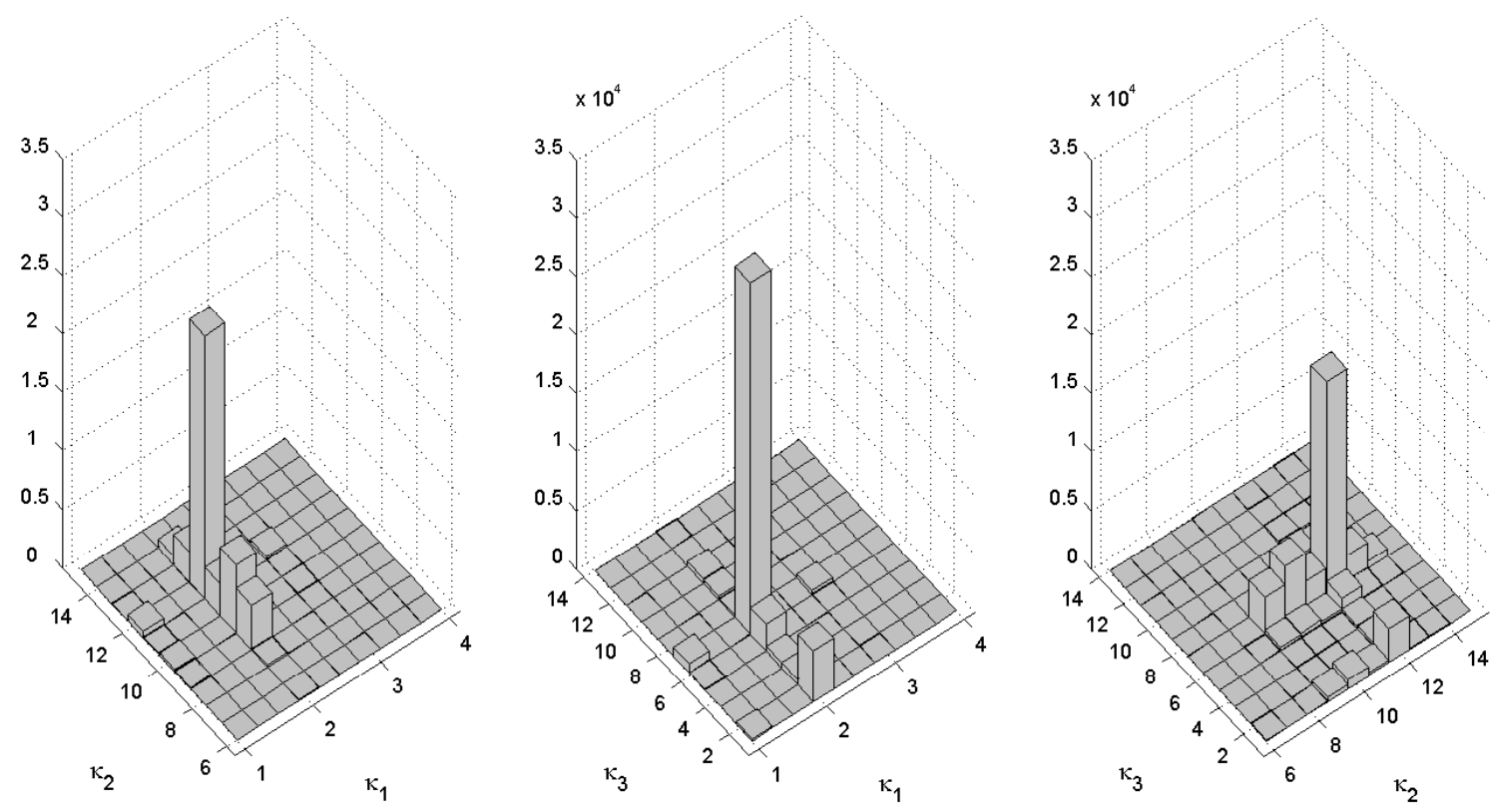

Note: The graphs show the posterior distribution of the lead time parameters $\kappa_{j}, j=1,2,3$, in the three regime MS-VAR model with imperfect synchronization with asymmetric (or regime-specific) phase shifts for industrial production (IP) and the Conference Board leading economic index (LEI), summarized in Table 6.

As before, the structural break in volatility is dated in February 1984. The complete posterior distribution for $\tau$ shows that this estimate is quite precise. Hence, the uncertainty about the timing of the Great Moderation is small.

Before closing this section, some remarks about a possible 'recovery' phase (instead of or in addition to a severe recession regime) in the US business cycle are in order. First of all, it is important to note that our model specification does not enforce the nature of the third (or any other) regime. The restrictions on the mean parameters in (4) pin down the regimes only in terms of rank order but not in terms of the sign of the mean growth rates. Hence our three regime model could also have resulted in identifying a recovery regime. In fact, we estimated three regime models 
with certain restrictions on the mean growth rates to force the model to describe a recovery phase (in addition to expansions and recessions). The estimation results (available upon request) show that, when imposed, a recovery regime occurs mostly following the recessions before 1990. This indicates that recoveries follow more severe recessions, suggesting that an extension of the model in that direction may be useful, see Morley and Piger (forthcoming) for various possibilities. Interestingly, the lead time of the LEI for the recovery regime is almost the same as for the expansion regime. Hence, it conveys limited information about the prediction of the end of recessions beyond the information embodied in the expansion regime. Moreover, this model resulted in poor predictive likelihood value, below that of the two and three regime models. Hence, we conclude that the model we present here explains the business cycle characteristics of IP and the LEI better than that model with a third recovery regime.

\section{Severe recessions and the theory of the financial accelerator}

The economic downturns identified in our previous analysis have in common that they all start as a mild recession. Differences occur as the downturn proceeds, in the sense that some recessions remain relatively mild while others turn into severe contractions. This empirical result establishes a link to economic theories about the severity of recessions. In particular, the finding that all recessions start with relatively small shocks but then differ in terms of the amplification of these adverse shocks and/or the magnitude of the following shocks is consistent with the theory of the 'financial accelerator'. This states that borrowers facing relatively high agency costs in credit markets will bear the brunt of economic downturns (due to the flight to quality mechanism). This suggests that credit spreads, that is the difference in 
yields on corporate bonds issued by firms with low and high credit quality, should widen during recessions. Furthermore, the increase in these spreads may be expected to be larger for deeper contractions. To examine this possibility, Figure 7 displays the posterior probabilities of the mild and severe recession regimes together with the (level and first difference of the) spread between Moody's Baa and Aaa corporate bond yields. Note that this spread should indicate the severity of the credit squeeze in extreme market conditions, as it measures the yield difference between two groups of relatively safe investment-grade bonds.

Figure 7 reveals an interesting pattern. The highest levels of the credit spread are clearly associated with the severe recessions identified in 1974-5, 1980 and 2007-9. Especially the most pronounced increases of the spread correspond to the start of the severe recession phases. The relation between the mild recession regime and the credit spread is much less clear. If an increase in the spread can be observed at all, it is very minor compared to the level shifts around severe recessions. The behavior of the credit spread clearly provides evidence of the relation between the financial accelerator and the third regime captured in our analysis. 
Figure 7: Posterior recession probabilities and Moody's Baa-Aaa credit spread
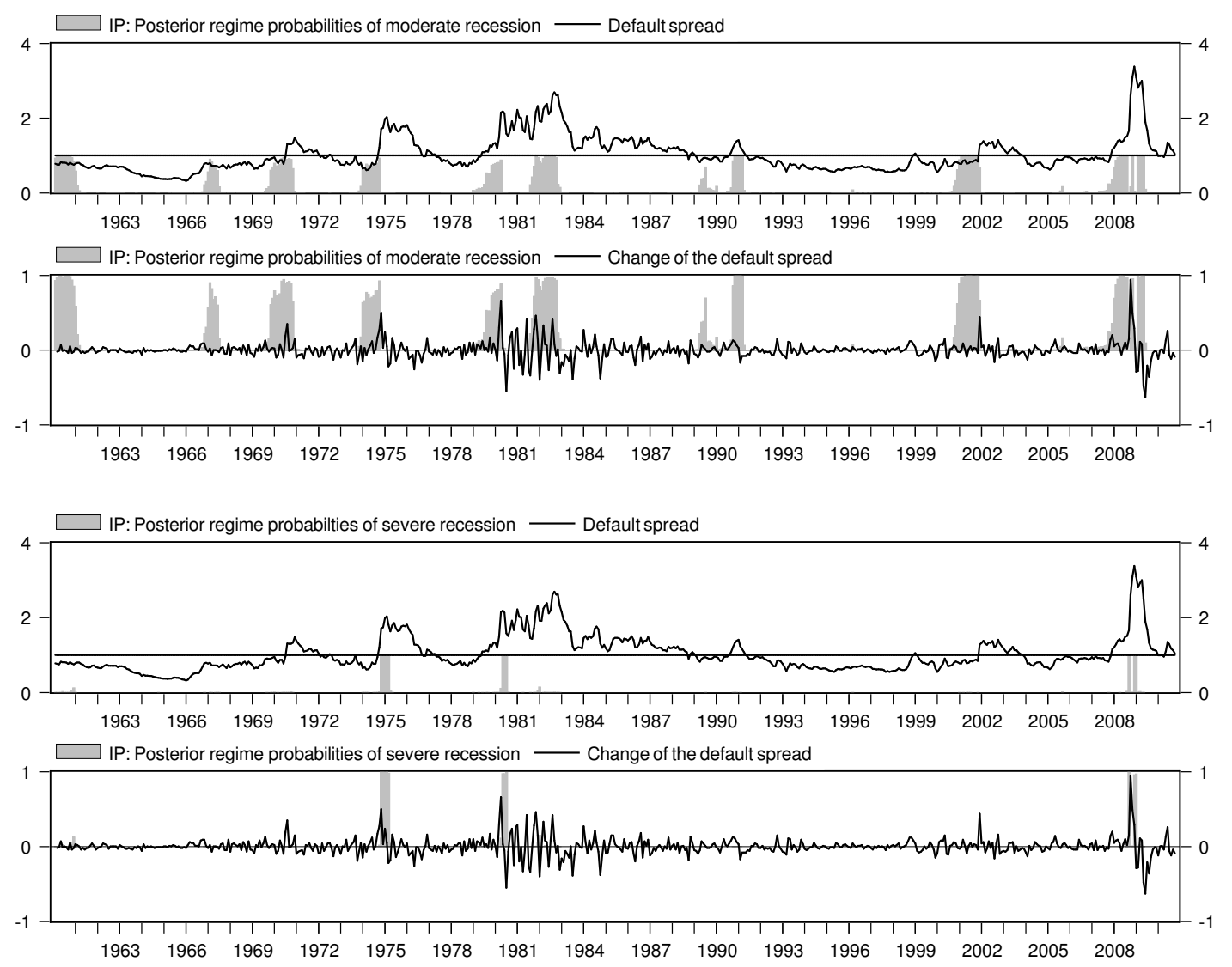

Note: The graphs show the posterior regime probabilities for mild and severe recessions in the three regime MS-VAR model with imperfect synchronization with asymmetric (or regime-specific) phase shifts for industrial production (IP) and the Conference Board leading economic index (LEI), summarized in Table 6, together with the level and first difference of the spread between Moody's Baa and Aaa corporate bond yields.

We further analyze this effect using more specific data on corporate bonds, comprising option-adjusted-spreads (OAS) of investment-grade corporate bonds with ratings $\mathrm{AAA}$ and $\mathrm{BBB}$ and high yield corporate bonds with ratings $\mathrm{CCC}$ and below. ${ }^{9}$

\footnotetext{
${ }^{9}$ This data represents the OASs of subsets of the Bank of America Merrill Lynch US Corporate Master Index, tracking the performance of US dollar denominated investment grade rated corporate debt publicly issued in the US domestic market. The BofA Merrill Lynch OASs are the calculated spreads between a computed OAS index of all bonds in a given rating category and a spot Treasury curve. The data is obtained from the FRED database of Federal Reserve Bank of St. Louis.
} 
As the benchmark yields for these spreads are mostly identical (namely treasuries with similar maturities), the differences between these OASs of corporate bonds with different credit ratings provide detailed measures of the credit risk premiums in the corporate bond market, which may provide more insight in the relevance of the financial accelerator effect for the severity of recessions. We compute three spreads, namely the spread between the high yield (CCC) corporate bonds and the investment-grade bonds with either AAA or BBB rating, as well as the spread between yields on BBB- and AAA-rated bonds. A limitation of this dataset is that it only dates back until January 1997. Still the period from January 1997 until October 2010 covers the two most recent recessions with distinct characteristics, hence, it may provide sufficient information for our analysis. We display the posterior regime probabilities of the mild and severe recession regimes together with these three spreads and their first differences in Figure 8.

The results confirm our previous analysis. The spread between the two groups of investment grade bond yields increases substantially around the severe recession of 2007-9, while it does not exhibit a clear departure from its path during the recession in 2001. Additionally, the spreads between the high-yield and investment-grade bonds (both $\mathrm{BBB}$ and AAA) increase in both of the recessions, albeit more pronounced during the severe recession. Put differently, the spreads between relatively risky and safe bonds always increase during recessions, as expected, but the magnitude (as well as the speed) of the changes is positively related to the severity of the recession. These two different degrees of disfunctioning of the credit channel and its reflection on the economy is nicely captured by our two different types of recession regimes. Our findings corroborate the conclusions of Claessens et al. (2009), who focus on the role of interactions between macroeconomic and financial variables in determining the severity and duration of a recession. Analyzing recessions worldwide their evidence indicates that severe and deep recessions mostly are triggered 
by (or at least coincide with) credit crunches and house price busts.

Figure 8: Posterior recession probabilities and credit spreads between corporate bonds of different quality
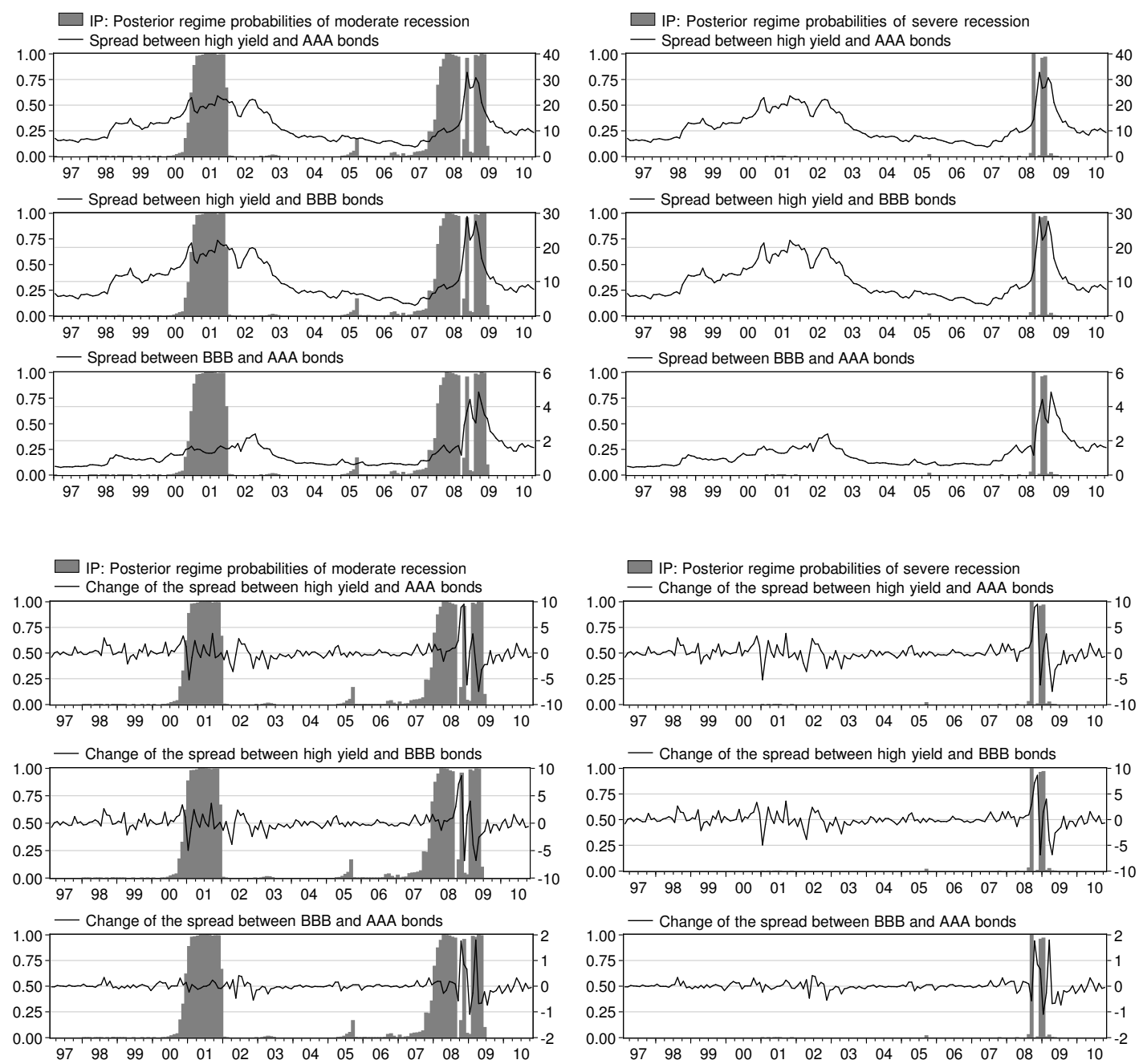

Note: The graphs show the posterior regime probabilities for mild and severe recessions in the three regime MS-VAR model with imperfect synchronization with asymmetric (or regime-specific) phase shifts for industrial production (IP) and the Conference Board leading economic index (LEI), summarized in Table 6, together with the level and first difference of credit spreads between high yield and investment grade corporate bonds. 


\section{Conclusion}

The macroeconomic experience during the post-World War II period has provided distinct evidence on US recessions. While recessions before the 1980s were short but relatively severe, subsequent recessions in 1990-1 and 2001 where shorter in duration but followed by sluggish recoveries. Interestingly, the most recent recession of 2007-9 appears to be unique in its characteristics in the sense that it comprises the worst parts of both previous types. Motivated by these observations, we provide empirical evidence for two distinct types of US recessions using Markov-switching models. First, using univariate analysis of monthly coincident and leading economic indicators we show that a three regime model renders a more accurate characterization of their business cycle regimes and dynamics than a model with two regimes only. Interestingly, the third regime captures 'severe recessions', contrasting the conventional view of a 'recovery' phase as the additional third regime.

This empirical finding is confirmed in a bivariate analysis of industrial production and the Conference Board's Leading Economic Index, using Markov-switching vector autoregressive models that allow for phase shifts between the cyclical regimes of these two variables. Results indicate that a three regime model with a severe recession phase (next to regimes for expansions and mild recessions) describes the data better than the two regime models with only recession and expansion regimes and a three regime model with a recovery phase. The occurrence of the severe recession regime mostly corresponds with periods of substantial credit squeezes and dramatic increases in credit spreads, providing evidence of the financial accelerator effect. 


\section{Appendix A Bayesian analysis}

In this appendix we describe the different aspects involved in the Bayesian analysis of Markov-switching models. We discuss the specification of prior distributions in Section A.1. This is followed by a derivation of the complete data likelihood function in Section A.2 and a description of the posterior simulator in Section A.3. Finally, we consider model selection based on the predictive likelihood in Section B.

We follow the common practice in the literature to treat the unobserved regimes in Markov-switching models as parameters to be estimated. For this purpose we derive the complete data likelihood function. Here we provide details for the bivariate MS-VAR model as given in (5)-(6), with imperfect synchronization due to asymmetric phase shifts as specified in (8) and covariance matrix as in (9)-(10). The likelihood for the model with symmetric phase shifts follows straightforwardly as it is nested in this general specification. For notational convenience, let $\mathcal{S}_{1, t}$ and $\mathcal{S}_{2, t}$ be the $J \times 1$ vectors of regime indicators for the first and second variable respectively. The elements in these vectors take the values 0 or 1 , indicating which of the $J$ regimes occurs at time $t$. We write the model (5) in vector notation

$$
Y_{t}-\mathcal{S}_{t}^{\prime} \mu=\Phi\left(Y_{t-1}-\mathcal{S}_{t-1}^{\prime} \mu\right)+\mathcal{E}_{t} \quad \mathcal{E}_{t} \sim N\left(0, \Sigma_{\mathcal{S}_{t}}\right)
$$

where $Y_{t}=\left(y_{1, t}, y_{2, t}\right)^{\prime}, \mathcal{E}_{t}=\left(\varepsilon_{1, t}, \varepsilon_{2, t}\right)^{\prime}, \Phi$ is a $(2 \times 2)$ matrix containing the autoregressive parameters and where

$$
\mathcal{S}_{t}=\left(\begin{array}{cc}
\mathcal{S}_{1, t} & \mathbf{0}_{J} \\
\mathbf{0}_{J} & \mathcal{S}_{2, t}
\end{array}\right),
$$

with $\mathbf{0}_{J}$ a $J \times 1$ vector of zeros. 


\section{A.1 Prior distributions}

As we want the data to drive our estimation results we specify diffuse prior distributions for the model parameters. The parameters of key interest are the lead/lag times or phase shifts $\kappa=\left(\kappa_{1}, \ldots, \kappa_{J}\right)$. We use a discrete uniform prior where we assign equal probability to each possible value of $\kappa$ in a predefined set $\mathcal{C}$, that is

$$
f(\kappa) \propto \begin{cases}1 & \text { for all } \kappa \in \mathcal{C} \\ 0 & \text { otherwise }\end{cases}
$$

The specification of the set $\mathcal{C}$ determines the type of synchronization that is assumed. For example, when $\mathcal{C}=\left\{\kappa \in \mathbb{Z}^{J} \mid-c_{j} \leq \kappa_{j} \leq c_{j}, j=1, \ldots, J\right\}$ for certain positive valued $c_{j}, j=1, \ldots, J$, we allow for imperfect synchronization with the phase shifts being restricted only by the bounds $c_{j}$. Additional restrictions may be imposed such that, for example, the difference between the phase shifts of the distinct regimes cannot exceed a certain threshold $d$, that is, $\mathcal{C}=\left\{\kappa \in \mathbb{Z}^{J}\left|-c_{j} \leq \kappa_{j} \leq c_{j},\right| \kappa_{i}-\kappa_{j} \mid \leq\right.$ $d, i, j=1, \ldots, J\}$. Note that setting $d$ equal to zero (while $c_{j}>0$ for $j=1, \ldots, J$ ) results in a model where phase shift parameters are identical across regimes as given in (7). Setting $c_{j}=0$ implies an MS-VAR model with perfect synchronization .

For the transition probabilities we use an uninformative Dirichlet prior

$$
p_{1 j}, \ldots, p_{J j} \sim \operatorname{Dir}(1, \ldots, 1)
$$

When this non-informative prior for the transition probabilities is used special attention must be paid to the prior specifications of the regime-dependent parameters. This follows from the fact that the value of the complete data likelihood function is the same if we switch all regime dependent parameters together with the corresponding transition probabilities. This 'label switching problem' complicates proper posterior analysis as the posterior distributions of the regime dependent parameters 
become multimodal, see Frühwirth-Schnatter (2001) and Geweke (2007) for discussion. To circumvent this problem, we define the prior for the regime dependent mean parameters $\mu=\left(\mu_{1}^{\prime}, \mu_{2}^{\prime}\right)^{\prime}$ in such a way that it identifies the regimes. Specifically, we set the prior specification for $\mu$ as

$$
f(\mu) \propto \begin{cases}1 & \text { if } \mu_{1} \in\left\{\mu_{1} \in \mathbb{R}^{J} \mid \mu_{1,1}<\ldots<\mu_{1, j}<\ldots<\mu_{1, J}\right\} \\ 0 \quad & \text { elsewhere. }\end{cases}
$$

For the regime-dependent variance parameters $\sigma^{2}=\left\{\sigma_{1, j}^{2}, \sigma_{2, j}^{2}\right\}_{j=1}^{J}$ as well as for the correlation parameter $\rho$ we take uninformative priors

$$
\begin{aligned}
f\left(\sigma_{l, j}^{2}\right) & \propto \sigma_{l, j}^{-2} \quad \text { for } l=1,2 \text { and } j=1, \ldots, J, \\
f(\rho) & \propto\left(1-\rho^{2}\right)^{-\frac{3}{2}}
\end{aligned}
$$

which correspond to the uninformative prior $|\Sigma|^{-3 / 2}$ for $\Sigma$ in case of constant variances. In case we assume a single structural break in the covariance structure, then the prior distribution of the structural break parameter $\tau$ has a discrete uniform distribution

$$
f(\tau)= \begin{cases}\frac{1}{T-1-2 b} & \text { if } \tau \in\{k+b+1, \ldots, T-b\} \\ 0 & \text { elsewhere }\end{cases}
$$

and hence we do not allow for a break in the first and last $b$ observations of the sample period. For the size of the break $\delta$ we use an uninformative prior

$$
f\left(\delta^{2}\right) \propto \delta^{-2}
$$

Finally, the prior for the autoregressive coefficients is flat on the stationary region, that is,

$$
f(\Phi) \propto 1
$$


when the characteristic roots of $\Phi$ lie outside the unit circle and 0 otherwise.

\section{A.2 Complete data likelihood function}

Given the assumption of multivariate normality for the shocks $\mathcal{E}_{t}$, the conditional density of $Y_{t}$ given the past observations $Y^{t-1}=\left\{Y_{1}, \ldots, Y_{t-1}\right\}$ and given the past and current states $S^{t}=\left\{S_{1}, \ldots, S_{t}\right\}$ is given by

$$
f\left(Y_{t} \mid Y^{t-1}, S^{t}, \mu_{1}, \mu_{2}, \sigma_{1}^{2}, \sigma_{2}^{2}, \rho, \tau, \delta, \Phi, \kappa\right)=\left(\frac{1}{\sqrt{2 \pi}}\right)^{2}\left|\Sigma_{t}\right|^{-\frac{1}{2}} \exp \left(-\frac{1}{2} \mathcal{E}_{t}^{\prime} \Sigma_{t}^{-1} \mathcal{E}_{t}\right)
$$

where $\mu_{l}=\left(\mu_{l, 1}, \ldots, \mu_{l, J}\right)^{\prime}$ and $\sigma_{l}^{2}=\left(\sigma_{l, 1}^{2}, \ldots, \sigma_{l, J}^{2}\right)^{\prime}$ for $l=1,2, \kappa=\left(\kappa_{1}, \ldots, \kappa_{J}\right)^{\prime}$. The complete data likelihood function for model (A.1) conditional on the first observation equals

$$
f\left(Y^{T}, S^{T} \mid Y_{1}, \theta\right)=\left(\prod_{i=1}^{J} \prod_{j=1}^{J}\left(p_{i j}^{T_{i j}}\right)\right) \prod_{t=2}^{T} f\left(Y_{t} \mid Y^{t-1}, S^{t}, \mu_{1}, \mu_{2}, \sigma_{1}^{2}, \sigma_{2}^{2}, \rho, \tau, \delta, \Phi, \kappa\right),
$$

where $T$ denotes the sample size, $T_{i j}$ is the number of transitions from regime $i$ to regime $j$, and $\theta=\left(\mu_{1}, \mu_{2}, \sigma_{1}^{2}, \sigma_{2}^{2}, \rho, \delta\right.$, vec $(\Phi), \kappa$, vec $\left.(P)\right)$ represents all the model parameters, with $P=\left\{p_{i j}\right\}_{i, j=1}^{J}$ the matrix with transition probabilities. The likelihood function conditional only on the model parameters can be obtained by summing (A.12) over all the possible states as

$$
f\left(Y^{T} \mid Y_{1}, \theta\right)=\sum_{S_{1,1}=1}^{J} \sum_{S_{1,2}=1}^{J} \cdots \sum_{S_{1, T}=1}^{J} f\left(Y^{T}, S^{T} \mid Y_{1}, \theta\right)
$$




\section{A.3 The Gibbs sampler and full conditional posterior dis- tributions}

\section{Gibbs sampler}

The simulation scheme of the Gibbs sampler is as follows

1. Sample $\mu$ from $f\left(\mu \mid \Phi, \sigma^{2}, \rho, \tau, \delta, S^{T}, Y^{T}\right)$.

2. Sample $\Phi$ from $f\left(\Phi \mid \mu, \sigma^{2}, \rho, \tau, \delta, S^{T}, Y^{T}\right)$.

3. Sample $\sigma_{l}^{2}$ from $f\left(\sigma_{l}^{2} \mid \mu, \Phi, \rho, \tau, \delta, S^{T}, Y^{T}\right)$ for $l=1,2$.

4. Sample $\rho$ from $f\left(\rho \mid \mu, \Phi, \sigma^{2}, \tau, \delta, S^{T}, Y^{T}\right)$.

5. Sample $\tau$ from $f\left(\tau \mid \mu, \Phi, \sigma^{2}, \rho, \delta, S^{T}, Y^{T}\right)$.

6. Sample $\delta$ from $f\left(\delta \mid \mu, \Phi, \sigma^{2}, \rho, \tau, S^{T}, Y^{T}\right)$.

7. Sample $P$ from $f\left(p_{i j} \mid S_{1}^{T}\right)$.

8. Sample $\kappa_{j}$ from $f\left(\kappa_{j} \mid \mu, \Phi, \sigma^{2}, \rho, \tau, \delta, S_{1}^{T}, Y^{T}\right)$ for $j=1, \ldots, J$.

9. Sample $S_{1}^{T}$ from $f\left(S_{1}^{T} \mid P, \mu, \Phi, \sigma^{2}, \rho, \tau, \delta, \kappa, Y^{T}\right)$.

\section{Sampling of $\mu$}

To sample $\mu$ we first rewrite (A.1) as

$$
\Sigma_{t}^{-\frac{1}{2}}\left(Y_{t}-\Phi Y_{t-1}\right)=\Sigma_{t}^{-\frac{1}{2}}\left\{\mathcal{S}_{t}^{\prime}-\Phi \mathcal{S}_{t-1}^{\prime}\right\} \mu+\Sigma_{t}^{-\frac{1}{2}} \mathcal{E}_{t}
$$

This is a multivariate regression in the form of

$$
Z_{t}=X_{t} \mu+\epsilon_{t} \quad \text { with } \epsilon_{t} \sim N\left(0, I_{2}\right),
$$


and hence the conditional distribution of $\mu$ is multivariate normal with mean $\left(X^{\prime} X\right)^{-1}$ $X^{\prime} Y$ and variance $\left(X^{\prime} X\right)^{-1}$, where $X=\left(X_{2}^{\prime}, \cdots, X_{T}^{\prime}\right)^{\prime}$ and $Z=\left(Z_{2}^{\prime}, \cdots, Z_{T}^{\prime}\right)^{\prime}$, see Zellner (1971). The restrictions on the elements of $\mu$ that are imposed for identification of the regimes can be applied by sampling from the corresponding truncated distribution or using acceptance rejection sampling.

\section{Sampling of $\Phi$}

Conditional on remaining model parameters the model in (A.1) is a multivariate regression of the form

$$
Z=\Phi X+U
$$

where $Z=\left(Z_{2}, \cdots, Z_{T}\right)$ with $Z_{t}=Y_{t}-\mathcal{S}_{t}^{\prime} \mu$, and $X=\left(X_{2}, \cdots, X_{T}\right)$ with $X_{t}=$ $\left(Y_{t-1}-\mathcal{S}_{t-1}^{\prime} \mu\right)$ and

$$
\Omega^{-1}=\operatorname{Cov}(\operatorname{vec}(U))^{-1}=\operatorname{diag}\left(\Sigma_{2}^{-1}, \cdots \Sigma_{T}^{-1}\right) .
$$

Using the fact that

$$
\operatorname{vec}(Z)=\left(X^{\prime} \otimes I_{2}\right) \operatorname{vec}(\Phi)+\operatorname{vec}(U)
$$

we can simply write the regression model as a univariate regression

$$
\mathbf{z}=\left(X^{\prime} \otimes I_{2}\right) \operatorname{vec}(\Phi)+\mathbf{u}
$$

It then follows that the conditional posterior distribution of $\operatorname{vec}(\Phi)$ is multivariate normal with mean $\left(\left(X^{\prime} \otimes I_{2}\right)^{\prime} \Omega^{-1}\left(X^{\prime} \otimes I_{2}\right)\right)^{-1}\left(\left(X^{\prime} \otimes I_{2}\right)^{\prime} \Omega^{-1} z\right)$ and covariance matrix

$\left(\left(X^{\prime} \otimes I_{2}\right)^{\prime} \Omega^{-1}\left(X^{\prime} \otimes I_{2}\right)\right)^{-1}$, where

$$
\Omega^{-1}=\operatorname{Cov}(u)^{-1}=\operatorname{diag}\left(\Sigma_{2}^{-1}, \ldots, \Sigma_{T}^{-1}\right)
$$


In case of zero restrictions on the elements of $\Phi$, one can easily modify $\left(X^{\prime} \otimes I_{2}\right)$ by dropping the corresponding columns of $\left(X^{\prime} \otimes I_{2}\right)$.

\section{Sampling of $P$}

From the conditional likelihood function (A.12), it follows that the transition probabilities from state $i$ can be written as

$$
f\left(p_{i, 1}, \ldots, p_{i, J}\right) \propto \prod_{j=1}^{J} p_{i, j}^{T^{i, j}} \quad \text { for } i=1, \ldots, J
$$

where $T^{i j}$ denotes the number of transitions from state $i$ to state $j$. This corresponds to the kernel of a Dirichlet distribution and hence the transition probabilities can be sampled from a Dirichlet distribution with parameters $T_{i j}$ for $j=1, \ldots, J-1$. The transition probability to state $J, p_{i J}$, follows from the restriction that $\sum_{j=1}^{J} p_{i j}=1$.

\section{Sampling of Lead/Lag Parameters $\kappa$}

As the $\kappa_{j}$ parameters can only take discrete values we can compute the posterior probabilities for all $\kappa \in \mathcal{C}$ and sample from a multinomial distribution. We can sample all $\kappa_{j}$ parameters at once or conditional on each other, one at a time. When the number of admissible $\kappa$ 's is large, the latter approach may be more attractive as the number of combinations increases only linearly in the number of states, whereas it increases exponentially in the first case.

\section{Sampling of Regimes}

The conditional posterior density of $S_{1, t}$ denoted by $f\left(S_{1, t} \mid S_{1}^{-t}, \theta, Y^{T}\right)$ for $t=1, \ldots, T$ and $S_{1}^{-t}=S_{1}^{T} \backslash\left\{S_{1, t}\right\}$, is proportional to the transition probabilities due to the Markov structure and to the density of $Y$ conditional on the regimes. Hence, we can 
write the posterior density of $S_{1, t}$

$$
f\left(S_{1, t} \mid S_{1}^{-t}, \theta, Y^{T}\right) \propto f\left(S_{1, t} \mid S_{1, t-1}, \theta\right) f\left(S_{1, t+1} \mid S_{1, t}, \theta\right) \prod_{i=t-\kappa_{\min }}^{t+1+\kappa_{\max }} f\left(Y_{i} \mid Y^{i-1}, S^{i}, \theta\right)
$$

where $f\left(Y_{t} \mid Y^{t-1}, S^{t}, \theta\right)$ is given in $(\mathrm{A} .11), \kappa_{\max }=\max \left(\kappa_{1}, \ldots, \kappa_{J}\right)$ and $\kappa_{\min }=$ $\min \left(\kappa_{1}, \ldots, \kappa_{J}\right)$. At time $t=T$ the term $f\left(S_{1, t+1} \mid S_{1, t}, \theta\right)$ drops out. The regime at $t=1$ can be sampled from the conditional distribution

$$
f\left(S_{1,1} \mid S_{1}^{-1}, \theta, Y^{T}\right) \propto f\left(S_{1} \mid \theta\right) f\left(S_{1,2} \mid S_{1,1}, \theta\right) \prod_{i=2}^{t+1+\kappa_{\max }} f\left(Y_{i} \mid Y^{i-1}, S^{i}, \theta\right)
$$

where the unconditional density $f\left(S_{1,1} \mid \theta\right)$ follows a multinomial distribution with ergodic probabilities of the Markov chain.

Sampling of the state variables can be implemented by starting from the most recent value of $S_{1}^{T}$ and sampling the states backward in time, one after another. After each step, the $t^{\text {th }}$ element of $S_{1}^{T}$ is replaced by its most recent draw.

\section{Sampling of $\tau$}

The conditional posterior density of $\tau$ is given by

$$
\begin{aligned}
f\left(\tau \mid s^{T}, \theta \backslash\{\tau\}, Y^{T}\right) & \propto \mathbb{I}[b+k+1<\tau \leq T-b] \\
& \times\left|\Sigma_{t}\right|^{-\frac{1}{2}(\tau+2)} \exp \left(-\frac{1}{2} \operatorname{tr}\left(\Sigma_{t}^{-1}\left(\sum_{k+1}^{\tau-1} \mathcal{E}_{t}^{\prime} \mathcal{E}_{t}\right)\right)\right),
\end{aligned}
$$

where $\Sigma_{t}$ is defined by (9)-(10). As $\tau$ can take discrete values on the range $[b+$ $k+1, T-b]$, we can sample from its full posterior distribution using the posterior probabilities of each of these discrete values. 


\section{Sampling of Variances and Correlations}

To sample the variances we decompose the multivariate normal distribution of vector of error terms in a conditional distribution of $\varepsilon_{2 t}$ given $\varepsilon_{1 t}$ and the marginal distribution of $\varepsilon_{1 t}$. This results in

$$
\begin{aligned}
\prod_{t=2}^{T} f\left(\mathcal{E}_{t}\right)= & \prod_{t=2}^{\tau-1} \frac{1}{\delta \sigma_{1, t}} \phi\left(\frac{\varepsilon_{1, t}}{\delta \sigma_{1, t}}\right) \frac{1}{\delta \sigma_{2, t} \sqrt{\left(1-\rho^{2}\right)}} \phi\left(\frac{\varepsilon_{2, t}-\rho \sigma_{2, t} / \sigma_{1, t} \varepsilon_{1, t}}{\delta \sigma_{2, t}\left(1-\rho^{2}\right)}\right) \\
& \prod_{t=\tau}^{T} \frac{1}{\sigma_{1, t}} \phi\left(\frac{\varepsilon_{1, t}}{\sigma_{1, t}}\right) \frac{1}{\sigma_{2, t} \sqrt{\left(1-\rho^{2}\right)}} \phi\left(\frac{\varepsilon_{2, t}-\rho \sigma_{2, t} / \sigma_{1, t} \varepsilon_{1, t}}{\sigma_{2, t}\left(1-\rho^{2}\right)}\right)
\end{aligned}
$$

where $\sigma_{l, t}^{2}$ denotes the variance of $\varepsilon_{l, t}, l=1,2$, which follows from (10) or (10) with $\sigma_{l, S_{l, t}}^{2}$ replaced by $\sigma_{l, t}^{2}$. To sample $\sigma_{1, j}^{2}$ we use a Metropolis-Hastings sampler (Metropolis et al., 1953; Hastings, 1970), where we use as candidate an inverted $\chi^{2}$ distribution with scale parameter $\sum_{t=2}^{\tau-1} I\left[S_{1, t}=j\right] \delta^{-2} \varepsilon_{1, t}^{2}+\sum_{t=\tau}^{T} I\left[S_{1, t}=j\right] \varepsilon_{1, t}^{2}$ and degrees of freedom $\sum_{t=2}^{T} I\left[S_{1, t}=j\right]$ for $j=1, \ldots, J$ which follows from the first part of the decomposition in (A.24) in combination with the prior. To sample $\sigma_{2, j}^{2}$ we use a similar approach where we consider the marginal of $\varepsilon_{2, t}$ in the decomposition.

To sample $\rho$ from its conditional posterior distribution we can again use (A.24), which leads to the following as

$$
\left(1-\rho^{2}\right)^{-3 / 2} \prod_{t=2}^{\tau-1} \frac{1}{\sqrt{\left(1-\rho^{2}\right)}} \phi\left(\frac{\varepsilon_{2, t}-\rho \sigma_{2, t} / \sigma_{1, t} \varepsilon_{1, t}}{\delta \sigma_{2, t}\left(1-\rho^{2}\right)}\right) \prod_{\tau}^{T} \frac{1}{\sqrt{\left(1-\rho^{2}\right)}} \phi\left(\frac{\varepsilon_{2, t}-\rho \sigma_{2, t} / \sigma_{1, t} \varepsilon_{1, t}}{\sigma_{2, t}\left(1-\rho^{2}\right)}\right) .
$$

We can easily implement the griddy Gibbs sampler approach of Ritter and Tanner (1992). Given that, $\rho \in(-1,1)$ we can setup a grid in this interval based on the precision we desire about the value of $\rho$.

(A.24) shows that the full conditional posterior distribution of $\delta^{2}$ is an inverted $\chi^{2}$ distribution with scale parameter $\sum_{t=2}^{\tau-1} \sum_{j=1}^{J} I\left[S_{1, t}=j\right] \sigma_{1, t}^{-2} \varepsilon_{1, t}^{2}+\sigma_{2, t}^{-2}\left(1-\rho^{2}\right)^{-2}\left(\varepsilon_{2, t}-\right.$ $\left.\rho \sigma_{2, t} / \sigma_{1, t} \varepsilon_{1, t}\right)^{2}$ and $2(\tau-1)$ degrees of freedom. Hence, we sample from this distribution for drawing $\delta$. 


\section{Appendix B Evaluation of Predictive Likelihood}

Selecting the best model is not an easy task when the competing alternatives embody regime-switching dynamics. In most cases standard testing procedures apply only when the number of regimes is the same in the models being compared. In this case marginal likelihood based comparisons, e.g. Bayes factors, can be implemented. When the task is determination of the number of the regimes, however, a complication arises when improper priors such as ours are used for the regime dependent parameters of interest. In this case, Bayes factors are not properly defined and tend to select the more parsimonious model (see Gelfand and Dey, 1994, for details). An alternative way of model comparison is by means of predictive Bayes factors computed using predictive likelihoods. This approach has the advantage that it is not affected by the choice of prior distributions and over-fitting, while it is directly related to the posterior model probabilities. Moving away from a two regime model to a model with higher number of regimes may increase the model fit, however, the resulting increase may simply because of model over-fitting brought by the additional regimes. If this is the case this would signal itself in the predictions of the model. In this sense, predictive likelihood evaluation provides also an immune methodology of model selection against over-fitting.

For a given model, the predictive likelihood of the observation at $t_{0}+1, Y_{t_{0}+1}$, conditional on the previous observations $Y^{t_{0}}$, is given by

$$
f\left(Y_{t_{0}+1} \mid Y^{t_{0}}\right)=\int f\left(Y_{t_{0}+1} \mid \theta\right) f\left(\theta \mid Y^{t_{0}}\right) d \theta
$$

where $p\left(\theta \mid Y^{t_{0}}\right)$ is the posterior distribution of the model parameters $\theta$ given the observations until $t_{0}$, and $p\left(Y_{t_{0}+1} \mid \theta\right)$ is the density of the observation $y_{t_{0}+1}$, which 
can be written as

$$
f\left(Y_{t_{0}+1} \mid \theta\right)=\sum_{j=1}^{J} f\left(Y_{t_{0}+1} \mid S_{1, t_{0}+1}=j, \theta\right) f\left(S_{1, t_{0}+1}=j \mid \theta, Y^{t_{0}}\right) .
$$

We can use the posterior simulator to obtain the distribution of the model parameters and estimate the predictive likelihood by $G^{-1} \sum_{g=1}^{G} f\left(Y_{t_{0}+1} \mid Y^{t_{0}}, \theta^{(g)}\right)$ where $G$ is a large number of draws from the posterior distribution. This can be extended to compute the predictive likelihood of a sequence $Y^{t_{0}+1, t_{0}+h}=\left(Y_{t_{0}+1}^{\prime}, \ldots, Y_{t_{0}+h}^{\prime}\right)^{\prime}$ where

$$
\left.f\left(Y^{t_{0}+1, t_{0}+h} \mid Y^{t_{0}}\right)=\int \prod_{l=1}^{h} f\left(Y_{t_{0}+l}\right) \mid Y^{T_{0}+l-1}, \theta\right) f\left(\theta \mid Y^{t_{0}}\right) d \theta
$$

see Fruhwirth-Schnatter (2006), for details. 


\section{References}

Barnard, J., R. McCulloch, and X. Meng (2000), Modeling Covariance Matrices In Terms Of Standard Deviations And Correlations, With Application To Shrinkage, Statistica Sinica, 10, 1281-1311.

Bernanke, B. (1981), Bankruptcy, Liquidity, and Recession, American Economic Review, 71, 155-59.

Bernanke, B., M. Gertler, and S. Gilchrist (1996), The Financial Accelerator and the Flight to Quality, The Review of Economics and Statistics, 78, 1-15.

Bernanke, B. S., M. Gertler, and S. Gilchrist (1999), The financial accelerator in a quantitative business cycle framework, in J. B. Taylor and M. Woodford (eds.), Handbook of Macroeconomics, vol. 1 of Handbook of Macroeconomics, chap. 21, Elsevier, pp. 1341-1393.

Board, C. (2001), Business Cycle Indicators Handbook, available at http://www . conference-board.org/publications/describebook. cfm?id=852.

Boldin, M. (1996), A Check on the Robustness of Hamilton's Markov Switching Model Approach to the Economic Analysis of the Business Cycle, Studies in Nonlinear Dynamics \& Econometrics, 1, 35-46.

Cakmakli, C., R. Paap, and D. van Dijk (2011), Modeling and Estimation of Synchronization in Multistate Markov-Switching Models, Discussion Paper 2011-002/4, Tinbergen Institute.

Claessens, S., M. A. Kose, and M. E. Terrones (2009), What Happens During Recessions, Crunches and Busts?, Economic Policy, 24, 653-700.

Clements, M. P. and H.-M. Krolzig (2003), Business cycle asymmetries: Character- 
ization and testing based on Markov-switching autoregressions, Journal of BusinessEGEconomic Statistics, 21, 196-211.

Fisher, I. (1933), The Debt-Deflation Theory of Great Depressions, Econometrica, $333-357$.

Frühwirth-Schnatter, S. (2001), Markov Chain Monte Carlo Estimation of Classical and Dynamic Switching and Mixture Models, Journal of the American Statistical Association, 96, 194-209.

Fruhwirth-Schnatter, S. (2006), Finite Mixture and Markov Switching Models, chap. 3.7.3, Springer Verlag, pp. 89-91.

Gelfand, A. E. and D. K. Dey (1994), Bayesian Model Choice: Asymptotics and Exact Calculations, Journal of the Royal Statistical Society. Series B (Methodological), 56, 501-514.

Geman, S. and D. Geman (1984), Stochastic Relaxations, Gibbs Distributions, and the Bayesian Restoration of Images, IEEE Transaction on Pattern Analysis and Machine Intelligence, 6, 721-741.

Gertler, M. and N. Kiyotaki (2010), Financial Intermediation and Credit Policy in Business Cycle Analysis, in B. M. Friedman and M. Woodford (eds.), Handbook of Monetary Economics, vol. 3 of Handbook of Monetary Economics, chap. 11, Elsevier, pp. 547-599.

Geweke, J. (2007), Interpretation and Inference in Mixture Models: Simple MCMC works, Computational Statistics \& Data Analysis, 51, 3529-3550.

Geweke, J. and G. Amisano (2007), Hierarchical Markov normal mixture models with applications to financial asset returns, Working Paper Series 831, European Central Bank. 
Geweke, J. and G. Amisano (2010), Comparing and Evaluating Bayesian Predictive Distributions of Asset Returns, International Journal of Forecasting, 26, 216-230. Gordon, R. J. (1993), The Jobless Recovery: Does It Signal a New Era of Productivity-led Growth?, Brookings Papers on Economic Activity, 24, 271-316.

Groshen, E. L. and S. Potter (2003), Has structural change contributed to a jobless recovery?, Current Issues in Economics and Finance.

Hamilton, J. D. (2005), What's Real About the Business Cycle?, NBER Working Papers 11161, National Bureau of Economic Research, Inc.

Hamilton, J. D. and G. Perez-Quiros (1996), What Do the Leading Indicators Lead?, Journal of Business, 69, 27-49.

Hastings, W. (1970), Monte Carlo Sampling using Markov Chains and their Applications, Biometrika, 57, 97-10.

Kim, C.-J., J. Morley, and J. Piger (2005), Nonlinearity and the Permanent Effects of Recessions, Journal of Applied Econometrics, 20, 291-309.

Kim, C.-J. and C. R. Nelson (1999), State-Space Models with Regime Switching: Classical and Gibbs-Sampling Approaches with Applications, vol. 1, 1 edn., The MIT Press.

Krolzig, H.-M. (1997), Markov-switching vector autoregressions, Springer Verlag, Berlin, New York.

McConnell, M. and G. Perez-Quiros (2000), Output Fluctuations in the United States: What Has Changed since the Early 1980's?, American Economic Review, 90, 1464-1476. 
Metropolis, N., A. Rosenbluth, M. Rosenbluth, A. Teller, and E. Teller (1953), Equations of State Calculations by Fast Computing Machines, Journal of Chemical Physics, 21, 1087-1092.

Mishkin, F. (1978), The Household Balance Sheet and the Great Depression, Journal of Economic History, 38, 918-937.

Morley, J. and J. Piger (forthcoming), The Asymmetric Business Cycle, The Review of Economics and Statistics.

Paap, R., R. Segers, and D. van Dijk (2009), Do Leading Indicators Lead Peaks More Than Troughs?, Journal of Business EG Economic Statistics, 27, 528-543.

Ritter, C. and M. A. Tanner (1992), Facilitating the Gibbs Sampler: The Gibbs Stopper and the Griddy-Gibbs Sampler, Journal of the American Statistical Association, $87,861-868$.

Romer, C. D. (1990), The Great Crash and the Onset of the Great Depression, The Quarterly Journal of Economics, 105, 597-624.

Romer, C. D. (1993), The Nation in Depression, Journal of Economic Perspectives, 7, 19-39.

Sensier, M. and D. van Dijk (2004), Testing for Volatility Changes in U.S. Macroeconomic Time Series, The Review of Economics and Statistics, 86, 833-839.

Sichel, D. E. (1994), Inventories and the Three Phases of the Business Cycle, Journal of Business \& Economic Statistics, 12, 269-77.

Tanner, M. A. and W. H. Wong (1987), The Calculation of Posterior Distributions by Data Augmentation, Journal of the American Statistical Association, 82, 528550. 
Zellner, A. (1971), An Introduction to Bayesian Inference in Econometrics, Wiley, New York. 